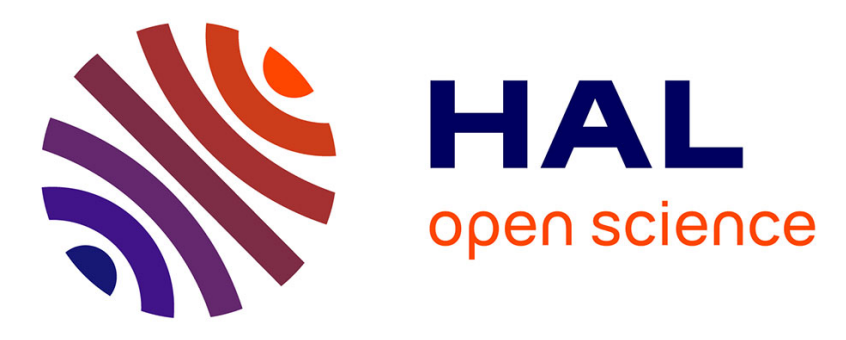

\title{
Efficient Lattice Boltzmann Solver for Patient-Specific Radiofrequency Ablation of Hepatic Tumors
}

Chloé Audigier, Tommaso Mansi, Hervé Delingette, Saikiran Rapaka, Viorel Mihalef, Daniel Carnegie, Emad Boctor, Michael Choti, Ali Kamen, Nicholas Ayache, et al.

\section{To cite this version:}

Chloé Audigier, Tommaso Mansi, Hervé Delingette, Saikiran Rapaka, Viorel Mihalef, et al.. Efficient Lattice Boltzmann Solver for Patient-Specific Radiofrequency Ablation of Hepatic Tumors. IEEE Transactions on Medical Imaging, 2015, 34 (issue 7 ), p 1576-1589. 10.1109/TMI.2015.2406575. hal-01146319

\section{HAL Id: hal-01146319 https://hal.inria.fr/hal-01146319}

Submitted on 28 Apr 2015

HAL is a multi-disciplinary open access archive for the deposit and dissemination of scientific research documents, whether they are published or not. The documents may come from teaching and research institutions in France or abroad, or from public or private research centers.
L'archive ouverte pluridisciplinaire HAL, est destinée au dépôt et à la diffusion de documents scientifiques de niveau recherche, publiés ou non, émanant des établissements d'enseignement et de recherche français ou étrangers, des laboratoires publics ou privés. 


\title{
Efficient Lattice Boltzmann Solver for Patient-Specific Radiofrequency Ablation of Hepatic Tumors
}

\author{
Chloé Audigier ${ }^{1,2}$, Tommaso Mansi ${ }^{2}$, Hervé Delingette ${ }^{1}$, Saikiran Rapaka ${ }^{2}$, Viorel Mihalef ${ }^{2}$, Daniel Carnegie ${ }^{4}$, \\ Emad Boctor ${ }^{3}$, Michael Choti ${ }^{5}$, Ali Kamen ${ }^{2}$, Nicholas Ayache ${ }^{1}$, Dorin Comaniciu ${ }^{2}$
}

\begin{abstract}
Radiofrequency ablation (RFA) is an established treatment for liver cancer when resection is not possible. Yet, its optimal delivery is challenged by the presence of large blood vessels and the time-varying thermal conductivity of biological tissue. Incomplete treatment and an increased risk of recurrence are therefore common. A tool that would enable the accurate planning of RFA is hence necessary. This manuscript describes a new method to compute the extent of ablation required based on the Lattice Boltzmann Method (LBM) and patient-specific, pre-operative images. A detailed anatomical model of the liver is obtained from volumetric images. Then a computational model of heat diffusion, cellular necrosis, and blood flow through the vessels and liver is employed to compute the extent of ablated tissue given the probe location, ablation duration and biological parameters. The model was verified against an analytical solution, showing good fidelity. We also evaluated the predictive power of the proposed framework on ten patients who underwent RFA, for whom pre- and post-operative images were available. Comparisons between the computed ablation extent and ground truth, as observed in postoperative images, were promising (DICE index: $42 \%$, sensitivity: $67 \%$, positive predictive value: $38 \%$ ). The importance of considering liver perfusion while simulating electrical-heating ablation was also highlighted. Implemented on graphics processing units (GPU), our method simulates 1 minute of ablation in $\mathbf{1 . 1 4}$ minutes, allowing near real-time computation.
\end{abstract}

Index Terms-Radio Frequency ablation, Patient-Specific Simulation, Lattice Boltzmann Method, Computational Fluid Dynamics, Heat Transfer, Therapy Planning

\section{INTRODUCTION}

\section{A. Clinical background}

In spite of recent advances in cancer therapy, treatment of primary and metastatic malignancies, including those in the liver, remains a significant challenge. Hepatocellular carcinoma (HCC) for example is one of the most common malignancies encountered throughout the world (more than 1 million

Copyright (c) 2010 IEEE. Personal use of this material is permitted. However, permission to use this material for any other purposes must be obtained from the IEEE by sending a request to pubs-permissions@iee.org.

chloe.audigier@inria.fr - Tel: +33 492387926 - 2004 route des Lucioles BP-93, 06902 Sophia-Antipolis Cedex, FRANCE

1 Inria Sophia-Antipolis, Asclepios Research Group, Sophia-Antipolis, France

${ }^{2}$ Siemens Corporate Technology, Imaging and Computer Vision, Princeton, NJ, USA

${ }^{3}$ Dept. of Radiology, Johns Hopkins Medical Institutions, Baltimore, MD, USA

4 Dept. of Surgery, Johns Hopkins Medical Institutions, Baltimore, MD, USA

5 Dept. of Surgery, UT Southwestern Medical Center, Dallas, TX, USA

Manuscript received April 19, 2005; revised December 27, 2012. cases per year), with increasing frequency in Western countries due to the changing prevalence of hepatitis C [1]. Unfortunately, less than $25 \%$ of patients with primary or secondary liver cancer are candidates for resection or transplantation, which are considered as the most effective treatments. These limitations are due to the patient's condition and the size, location, or number of the tumors. Consequently, minimally invasive ablative therapies such as radiofrequency ablation (RFA), high intensity focused ultrasound (HIFU), cryotherapy, microwave tumor coagulation and laser ablation have raised increasing interest for the treatment of liver tumors. Amongst them, RFA is the most widely used approach [2], but it is not yet a reliable alternative in clinical routine. There is a need for training tools for the less-experienced clinicians to improve outcome rates since the success rate increases quickly with experience [3]. During RFA procedure, the clinician places one or more probes percutaneously or during open surgery within the target area in the liver parenchyma. From the electrodes at the tip of the probe, a high frequency alternating electric current flows through the surrounding region and heating is induced due to the electric resistance of the living tissue. This results in thermal coagulative necrosis at temperatures above $50^{\circ} \mathrm{C}$ due to irreversible protein denaturation of the cells. Unlike extirpative therapies, the RFA treatment is difficult to monitor in vivo: success of the procedure depends on the complete coverage of the tumor by the thermal zone, which relies on optimal probe placements and the extent of conductive heat delivery. However, the latter is challenged by the hepatic blood vessels and the parenchyma perfusion that dissipate heat and make the size and shape of the ablation zone difficult to control thus potentially reducing RFA efficiency and increasing risks of recurrence [4]. These are the reasons why planning the RFA protocol for a specific patient is a challenging task and mathematical modeling has the potential to assist the radiofrequency ablation of tumors.

\section{B. Technical Background}

First, several computational models of RFA are presented. All these models are divided in 3 different modeling parts: an electrical heating model, a heat transfer in living tissue model, and a cellular necrosis model. These models are coupled as illustrated on Fig 1 and described in the following sections.

1) Computational Models of RFA: The traditional method to discretize RF ablation PDE is the Finite-Element Method 


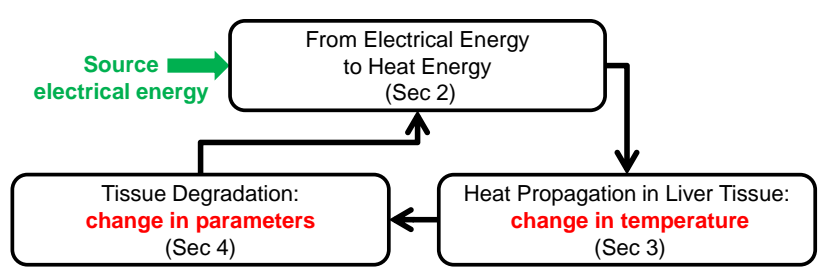

Fig. 1: Sketch of the coupled modeling steps of the biophysical RFA model.

(FEM). Studies coupling radiofrequency electrical fields to thermal transport have been developed to compute heat diffusion in the liver, predict the temperature distribution during the procedure and finally evaluate the optimal placement of the RFA probes [5]-[7]. A weighted distance-based method has been proposed to give a fast GPU-based real-time approximation of the ablation zone [8], but the validation is still an active area of research. Furthermore, a preprocessed thermal equilibrium representation of the liver parenchyma and blood vessels is needed to incorporate its cooling effect. However, to the best of our knowledge, none of these models rely on patient-specific data. The vascular system of the liver has to be considered but it is often neglected or simplified in these studies and blood flow circulation is not computed based on patient-specific clinical information. It is also particularly important to take into account the effect of perfusion, which is neglected in these models. Moreover, the use of classic FEM is often more computationally demanding than using recent numerical methods such as the Lattice Boltzmann Method (LBM) [9].

2) Models of Electrical Heating: Different types of radiofrequency (RF) probe can be used, either the cool-tip single probe or the RITA probe (StarBurst Radiofrequency Ablation, AngioDynamics, Latham, NY; www.angiodynamics.com) with three, four or six umbrella shaped prongs which can be deployed within the tumor (Fig. 2). In the literature, both probe configurations are simulated [10], [11]. The temperature and the voltage are usually set to be constant numbers on the surface of RF electrode probe needle and the potential field generated around the probe is solved numerically, using the Laplace equation for the electrical field in conductive media [12]. Even if this approach is theoretically accurate, it requires a fine volume mesh on the surface of the very thin probe tips and then suffers from a high computational cost. Furthermore, the exact position of the whole electrode probe is not always available and the imperfect needle positioning has been found to severely affect the outcome of RFA procedure [13]. The electric field strength is not high, most of the Joule effect heating is generated within the space $1 \mathrm{~mm}$ around the electrode surface [11] and most of the ablation zone at distances farther from the electrode is created by thermal conduction [14]. Moreover, the particular details of the heating point sources appear to have only a limited effect on the final lesion size [15]. Thus, in our framework, the electrical heating is modeled with a Dirichlet boundary conditions on a sphere, whose radius is defined pre-operatively by the protocol followed by the clinician.

3) Biophysical Models of Heat Transfer in Tissue: Computing heat diffusion in biological tissues amounts to solving the coupled bioheat equations derived from the theory of porous media (Eq. 1), where each elementary volume is assumed to comprise both tissue and blood with a certain fraction [16].

$$
\begin{aligned}
& (1-\epsilon) \rho c_{t i} \frac{\partial T_{t i}}{\partial t}=\underbrace{(1-\epsilon) Q}_{\text {source }}+(1-\epsilon) \underbrace{\nabla \cdot\left(d \nabla T_{t i}\right)}_{\text {diffusion }}+H\left(T_{b l}-T_{t i}\right) \\
& \epsilon \rho c_{b l}(\frac{\partial T_{b l}}{\partial t}+\underbrace{\mathbf{v} \cdot \nabla T_{b l}}_{\text {advection }})=\epsilon Q+\epsilon \nabla \cdot\left(d \nabla T_{b l}\right)-H\left(T_{b l}-T_{t i}\right)
\end{aligned}
$$

In theses equations, $T, Q, \mathbf{v}, \epsilon$ stand for temperature, source term, blood velocity, blood volume fraction (fraction of blood volume over total volume) and subscripts 'ti' and 'bl' refer to tissue and blood phase respectively. The other parameters are listed in Table I. Different models similar to (Eq. 1) have been proposed in the literature [17]-[20]. Some models include a metabolic heat generation term [21], which is neglected here since it has been shown to be insignificant in thermal ablation [22]. The advection term in the blood bioheat equation (Eq. 1b) is sometimes simplified by considering an averaged perfused tissue volume [20] but we preserve it in our model as it is essential when modeling the heat transfer between the tissue and the small vessels with low blood velocity magnitude. To account for the heat transfer associated with the transcapillary fluid exchange, some models include an additional perfusion heat transfer term [16]. However, it has been proven that this term can be neglected as the blood in the capillary network usually reaches equilibrium with tissue temperature [23]. Some bioheat models examined countercurrent heat transfer in arterial-venous vessels (the venous flow is warmed through heating from the nearby arteries) [17], [23], [24]. One common simplification of the coupled bioheat equations is the Pennes model [25] where the blood temperature is assumed constant, which is valid within and close to large vessels, where the blood velocity magnitude is high. In this case, the coupled bio-heat equations (Eq. 1) reduce to one unique equation with only one temperature distribution $T$ describing the temperature inside the liver:

$$
(1-\epsilon) \rho c_{t i} \frac{\partial T}{\partial t}=(1-\epsilon) Q+(1-\epsilon) \nabla \cdot(d \nabla T)+H\left(T_{b 0}-T\right)
$$

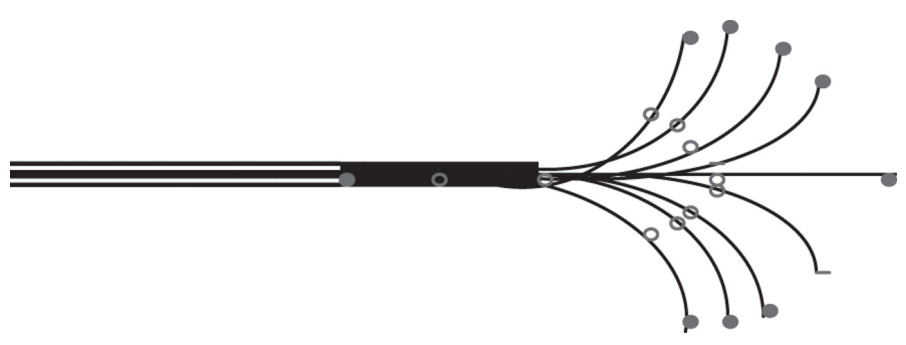

Fig. 2: RITA probe representation from [15]. 
TABLE I: Values from literature [15] of the parameters used in the computation

\begin{tabular}{|c|c|c|}
\hline parameter & description & value \\
\hline$\rho$ & blood and tissue densities & $1.06 \times 10^{3} \mathrm{~kg} \mathrm{~m}^{-3}$ \\
\hline$c_{b l}$ & blood heat capacity & $4.18 \times 10^{3} \mathrm{~J}(\mathrm{~kg} \mathrm{~K})^{-1}$ \\
\hline$c_{t i}^{A}$ & tissue heat capacity & $3.6 \times 10^{3} \mathrm{~J}(\mathrm{~kg} \mathrm{~K})^{-1}$ \\
\hline$c_{t i}^{D}$ & tissue heat capacity in dead cells & $0.67 \times 10^{3} \mathrm{~J}(\mathrm{~kg} \mathrm{~K})^{-1}$ \\
\hline$d$ & blood and tissue heat conductivities & $0.512 \times(1+0.00161 \times(T-310)) \mathrm{W}(\mathrm{m} \mathrm{K})^{-1}$ \\
\hline $\mathrm{H}$ & convective transfer coefficient & $24.4 \times 10^{5} \mathrm{~W}\left(\mathrm{~m}^{3} \mathrm{~K}\right)^{-1}$ \\
\hline$\epsilon$ & blood volume fraction & 0.1 \\
\hline$\kappa$ & permeability & $4.0 \times 10^{-11} \mathrm{~m}^{2}$ \\
\hline$\mu$ & dynamic viscosity of the blood & $0.0035 \mathrm{Pas}$ \\
\hline$\varphi_{v c_{i n}}$ & vena cava inflow & $2.0 \mathrm{~L} \mathrm{~min}^{-1}$ \\
\hline$\varphi_{i}$ & flow through the inlets of the hepatic veins & $1.6 \mathrm{~L} \mathrm{~min}^{-1}$ \\
\hline$p_{0}$ & vena cava outlet pressure & $3 \mathrm{mmHg}$ \\
\hline$k_{f}$ & forward rate constant & $3.33 \times 10^{-3} \mathrm{~s}^{-1}$ \\
\hline$k_{b}$ & backward rate constant & $7.77 \times 10^{-3} \mathrm{~s}^{-1}$ \\
\hline$T_{k}$ & parameter of cell state model & $40.5^{\circ} \mathrm{C}$ \\
\hline
\end{tabular}

This simplification has been used widely in the literature to model the electrical-thermal heating process happening in RFA. However, this model assumes that the blood is a volumetric heat sink and that it is uniformly distributed throughout the tissue [26]. Hence the term accounting for heattransfer convection between tissue and blood in the Pennes equation is oversimplified. This simplification may be suitable for a low vascularized organ like the heart and so it can be used more accurately to model cardiac ablation [27], [28] but it is not appropriate for the liver which is highly vascularized. Therefore, we have to account for the perfusion in the parenchyma in another way. Studies [26] demonstrate that microvasculature perfusion does not act as a spatially homogeneous heat sink, which invalidates the fundamental assumption of the Pennes model when applied to the liver in which different types of vessels are present. For these reasons another simplification has been proposed, which results in the Wulff-Klinger (WK) model [29], [30]. It assumes equilibrium between tissue and blood temperature which is accurate for highly perfused organ with small vessels where the blood velocity magnitude is low and accounts for the directional effect of the blood flow, with a blood volume fraction $\epsilon \ll 1$ :

$(1-\epsilon) \rho c_{t i} \frac{\partial T}{\partial t}=(1-\epsilon) Q+(1-\epsilon) \nabla \cdot(d \nabla T)-\epsilon \rho c_{b l} \mathbf{v} \cdot \nabla T$

This equation holds in a porous medium where tissue is dominating. The main difference between these two models lies in their cooling terms (last term of the right-hand side). The former is a reaction term and acts as a volumetric homogeneous heat sink whilst the latter is an advection term accounting for the directional effect of blood flow on the tissue temperature.

4) Models of Cellular Necrosis: Thermal treatments aim at transporting heat energy within the cancerous tumor then creating a zone of dead cells surrounded by tissue which could eventually recover after the ablation procedure. For a cell to go to an apoptosis state, a critical temperature should be exceeded during a sufficient time duration, due to the thermal tolerance of cells. Various cellular necrosis models [31]-[33] have been studied. The simplest one is to use a single temperature threshold above which cells stop instantaneously functioning, and below which cells remain fully functional. Although different values have been used for this threshold, the in vivo lesion volume (i.e. necrotic tissue after RF ablation) can be defined by the volume enclosed by the $50{ }^{\circ} \mathrm{C}$ isothermal surface [11], which has been widely used for RFA computational simulations. This isothermal model does not take into account the duration for which cells are at high temperatures although this factor has an influence on the cellular response since tissue damage is a function of both temperature and time [33]. Arrhenius-based models have been proposed [34], [35], which extend the Arrhenius law proposed for chemical reaction rates. The rate of cell damage is then proportional to $\exp (-E a / R T)$, where $E a$ is an activation energy, $R$ is the universal gas constant, and $T$ is the temperature. In this case, the tissue damage increases linearly with time and hyperbolically with temperature. Celldeath models based on the Arrhenius law are widely used, but are limited since the interpretation of model parameters characterizing the cell damage formulation is not easy to find. They are sensitive to small changes in parameters, and they are not able to predict cellular injury over a wide hyperthermic temperature range and throughout the entire heating process. To tackle these drawbacks, two-compartment models have been proposed containing either fully alive or fully dead cells. In order to characterize the biological state changes, transitions between the two compartments are usually modeled with firstorder rate processes. Finally a three-compartment cell death model [33] can be used to simulate both fast and slow cell death over a temperature range extending to $100^{\circ} \mathrm{C}$. This last model uses a single continuous function where a backward, recovery process is accounted for by adding a vulnerable state of the cell from which cells can either die or heal and return to the alive compartment. In [27], the authors define cumulative exposure as a measure of induced tissue injury. Cumulative exposure is the area under the temperature-time curve calculated on a voxel-basis over the duration of the ablation, which takes into account the voxel exposure prior to reaching the cell-death temperature (i.e., reversible damage and lesion penumbra) and also the voxel exposure beyond celldeath temperature for $5 s$ or longer (i.e., irreversible damage 


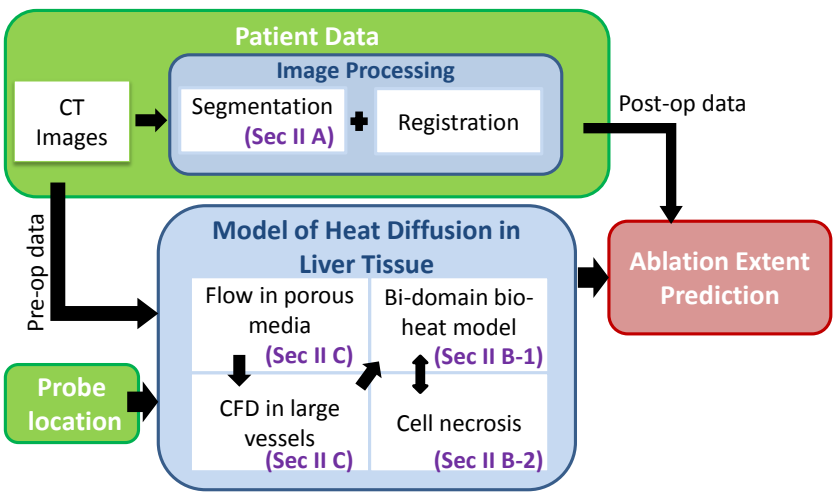

Fig. 3: Steps of the proposed method (green: input, blue: processes, red: output).

and core lesion). Their proposed exposure criterion yields consistent results with Arrhenius and the $50^{\circ} \mathrm{C}$ isotherm tissue injury criteria but at a lower computational expense.

\section{Aim of the Study}

Turning computational models of RFA into clinical practice remains challenging. The lack of an integrated, efficient, patient-specific framework for RFA modeling based on patient data is the major difficulty encountered. Studies based on in vivo animal images have been reported, but time-consuming FEM computations are still required [15]. Because of the expensive computation time needed by FEM, current approaches cannot enable a personalization of the model-based planning of RFA in a clinical setting. Therefore a fast and efficient model would help to optimize the treatment protocol preoperatively.

As a first step towards efficient patient-specific planning of RFA, we propose an integrated multiphysics approach that combines computational fluid dynamics (CFD) methods and Darcy's law with a biophysical model of the heat transfer and cellular necrosis to simulate RFA therapies in patients and predict the extent of ablation. This paper presents for the first time the Lattice Boltzmann Method (LBM) for heat in biological tissue. This method is a kinetic-based approach for fluid flow computations which has been successfully used as an alternative numerical method for solving Navier-Stokes type equations [36]. After a detailed convergence analysis against an analytical solution of the heat transfer equation in Sec. III-A and a computational efficiency study in Sec. III-B, we describe the application of our framework on fourteen tumors from ten patients with different hypotheses in Sec. III-C. The comparison with the real outcome extracted from postoperative data in terms of necrotic area suggests a promising correlation between the predicted and actual ablation extent, but also the importance of considering the blood perfusion inside the parenchyma. The generalization of the biophysical model with respect to tissue properties (i.e. we simulated a RFA procedure by using patient-specific geometry and boundary conditions but generic tissue parameters) shows that reasonable results can be achieved (average values of point-to-mesh distance: $10.17 \pm 8.52 \mathrm{~mm}$, DICE: $41.8 \%$, sensitivity: $66.9 \%$, positive predictive value: $38.3 \%$ ). This study extends our previous work [37] with a more detailed convergence analysis of the solution, the addition of a computational efficiency study, the generalization of the biophysical model with respect to tissue properties tested on ten patients data, and an analysis of the perfusion effect on the necrotic area. A first experiment of tissue parameters fitting has been performed on one patient. It highlighted a more accurate prediction power but also the need for personalization.

\section{MethodS}

As illustrated in Fig. 3, and detailed in the following section, we first estimate a comprehensive level set representation of the liver, including parenchyma, blood vessels and tumor from medical CT images. Then a computational model of heat propagation, cellular necrosis and blood flow through the vessels and liver is solved to estimate the extent of the ablated tissue. Algo. 1 presents the RFA computation method.

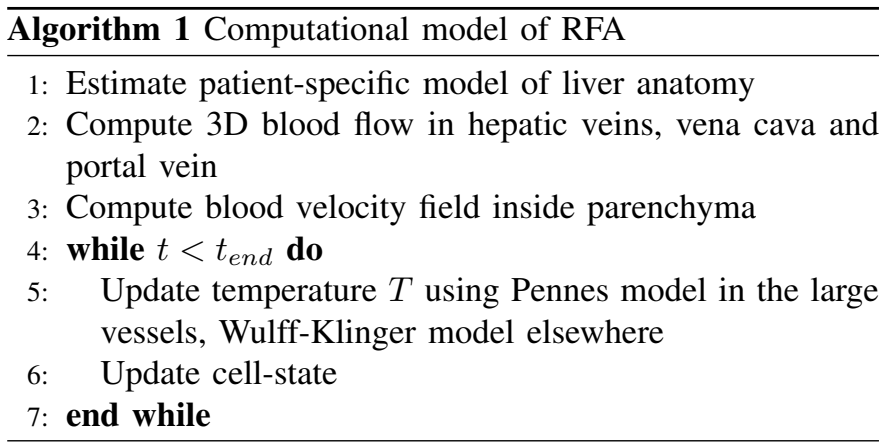

\section{A. Estimation of Anatomical Model from Patient Images}

For each patient, pre- and post-operative late venous phase CT are available. The images are semi-automatically segmented, yielding a detailed anatomical model of the patients liver. The user provides different strokes for the background and the foreground of the image, and the strokes can be modified interactively [38]. Finally the resulting segmentation is manually refined using itk-snap [39]. We generate volumetric binary images of the parenchyma, tumors, hepatic veins, vena cava, portal vein without the hepatic artery since only singlephase CT images are available (Fig. 4). The duration of the full segmentation process depends on the extent of the visible vessels. For example, typically for one patient, it took $20 \mathrm{~min}$ to segment the liver mask, $10 \mathrm{~min}$ for the tumor, $35 \mathrm{~min}$ for the vena cava and $15 \mathrm{~min}$ for the portal vein, i.e 1 hour 20 minutes in total. A smooth polygonal surface mesh is created for each region. A multi-label mask image is also created to identify the structures of interest for the computation.

\section{B. Fully Coupled Model of Heat Transfer in Liver Tissue and Cellular Necrosis}

1) Model of Heat Transfer in Liver Tissue: As current imaging techniques do not support an accurate measurement of the ratio between blood and liver tissue (large vessels can be clearly identified, but small capillaries are difficult to image), we use two simplifications of the coupled bioheat equation 


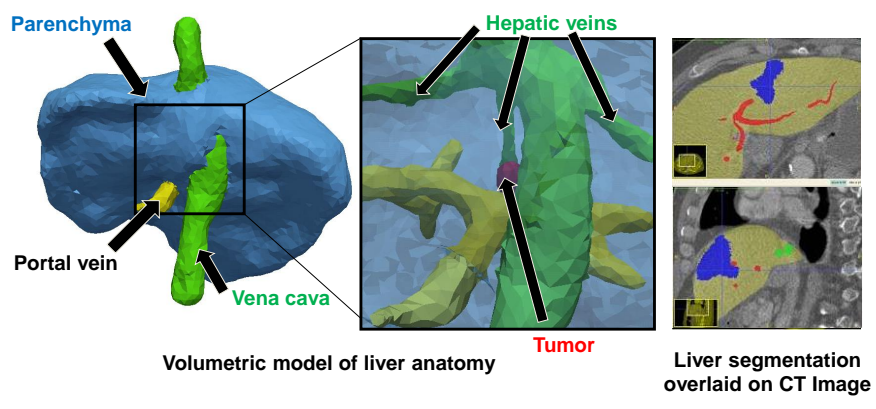

Fig. 4: A detailed anatomical model of the liver is estimated from a standard clinical CT image. See text for details.

(Eq. 1) in the parenchyma and in visible blood vessels. Both equations can be easily implemented in a modular way to cope with tissue inhomogeneity. Continuity between the two models has to be ensured at the extremities of the large vessels. To that end, $H$ is chosen large enough in order to reduce the effect of the high temperatures relative to normal body temperature, i.e $37^{\circ} \mathrm{C}$. In our framework, a two-compartment model is used according to the spatial location within the anatomy: either the Pennes model or the WK model is used. Assuming that large blood vessels and surrounding tissue are isolated from each other, we compute the temperature by solving the diffusion equation:

$$
\rho c_{t i} \frac{\partial T}{\partial t}=Q+\nabla \cdot(d \nabla T)
$$

everywhere in the domain, to which we add the cooling term:

$H\left(T_{b 0}-T\right) /(1-\epsilon)$ when a point belongs to a large vessel (Pennes model) or $-\epsilon \rho c_{b l} \mathbf{v} \cdot \nabla T /(1-\epsilon)$ when it belongs to the parenchyma (WK model).

2) Cellular Necrosis Model: Tissue necrosis is calculated based on the computed temperatures using a three-state model [33]. The variation in the concentration of alive (A), vulnerable (V) and dead (D) cells over time is computed according to the state equation (Eq. 5). We solve the resulting three coupled ODEs with a first order explicit scheme at each vertex of a Cartesian grid, yielding a spatially-varying cell state field used in the bio-heat solver. During the computation, the heat capacity is updated according to the state of the cell: $c_{t i}^{A}$ for alive or damaged cells, $c_{t i}^{D}$ for dead cells.

$$
\mathrm{A} \underset{k_{b}}{\stackrel{k_{f}(T)}{\longrightarrow}} \mathrm{V} \stackrel{k_{f}(T)}{\longrightarrow} \mathrm{D}
$$

$k_{f}(T)=\bar{k}_{f} e^{T / T_{k}}(1-A)$ and $k_{b}$ are the rates of cell damage and recovery respectively. $\overline{k_{f}}$ is a scaling constant and $T_{k}$ is a parameter that sets the rate of the exponential increase with respect to the temperature. A single damage process incorporates all physiological damage mechanisms, thus the transitions $\mathrm{A} \rightarrow \mathrm{V}$ and $\mathrm{V} \rightarrow \mathrm{D}$ are the same. The vulnerable state is an arbitrary position representing the "point of no return", it is not a change in the mechanism of thermal damage [33]. The initial conditions are chosen as in [33]: $A=0.99, V=0.01$ and $D=0$. To avoid stagnation in its initial condition due to the formula of $k_{f}(T)$, an initial fraction of the cells has to be in the vulnerable compartment. One percent seems reasonable since very small RMS error is achieved in [33] indicating a high quality of model fit to experimental data.

3) Numerical Resolution using LBM: We rely on the Lattice Boltzmann Method (LBM) where a statistical description of the system is used to compute heat diffusion and cellular necrosis in the liver tissue. LBM is a new computational method, which discretizes the velocity space on a grid, with mass, momentum and energy conservation conditions. To meet these three conservation conditions, two separate distribution functions are usually used [40]. In our approach, FEM solvers (CFD solver in the large vessels and porous solver in the parenchyma) give the blood flow distribution in the liver and enforce the mass and momentum conservation. Energy conservation is modeled using a distribution function for the thermal energy. LBM has emerged as a powerful technique for efficient computation of second order elliptic partial-differential equations [41]. It is used in Computational Fluid Dynamics to solve Navier-Stokes equations [36], in heat transfer problem [42], or in cardiac electrophysiology [9] to solve reaction-diffusion equations. Contrary to FEM, this discretization method uses a grid, allowing an easy parallelisation. We used an isotropic Cartesian grid and, LBM is performed with a D3Q7 scheme (DnQm denotes $\mathrm{m}$ discrete velocities in $\mathrm{n}$ dimensions): 6 directions are considered + the current point [9] as described in Fig 5, right. Numerically, LBM uses fictitious particles, which perform consecutive collision and streaming processes over the discrete lattice mesh. When there is a non-zero probability that particle distributions move to the same node from different directions, then the probability of having particle distributions at that node with a given velocity direction is changed due to the application of a collision operator. The governing equation at position $\mathbf{x}=(x, y, z)$ for the direction defined by the vector $\mathbf{e}_{i}$ is given by the two equations (Eq. 6, Eq. 7). $\mathbf{f}(\mathbf{x})=\left\{f_{i}(\mathbf{x})\right\}_{i=1 . .7}$ is the vector of the temperature energy distribution function with $f_{i}(\mathbf{x})$, the probability of finding a particle traveling along the vector $\mathbf{e}_{i}$ of the node $\mathbf{x}$ at a given time. $\hat{\mathbf{f}}(\mathbf{x})$ describes the post-collision distribution and $\boldsymbol{\omega}=\left\{\omega_{i}\right\}_{i=1 . .7}=(1 / 4,1 / 8,1 / 8,1 / 8,1 / 8,1 / 8,1 / 8)$ the vector of weighting factors [9] that depends on the lattice connectivity.

First, the collision step is:

$$
\begin{aligned}
& \hat{\mathbf{f}}(\mathbf{x}, t+\delta t)=\mathbf{f}(\mathbf{x}, t) \\
& +\mathbf{M}^{-\mathbf{1}} \hat{\mathbf{S}}\left[\mathbf{M} \mathbf{f}^{e q}(\mathbf{x}, t)-\mathbf{M f}(\mathbf{x}, t)\right]+\boldsymbol{\omega} \delta t H\left(T_{b 0}-T(\mathbf{x}, t)\right)
\end{aligned}
$$

and then, the streaming step is:

$$
f_{i}\left(\mathbf{x}+\mathbf{e}_{\mathbf{i}} \delta x, t+\delta t\right)=\hat{f}_{i}(\mathbf{x}, t+\delta t) \forall i \in 1 \ldots 7
$$

with:

$$
f_{i}^{e q}(\mathbf{x}, t)=\omega_{i} T(\mathbf{x}, t)\left[1+\frac{\mathbf{e}_{\mathbf{i}} \cdot \mathbf{v}}{c c_{s}^{2}}\right]
$$

We denote $c=\delta x / \delta t, c_{s}^{2}=1 / 4$ and $\delta x$ as the spacing. The set of vectors $\mathbf{e}_{i}$ is defined as:

$$
\left[\mathbf{e}_{1}, \mathbf{e}_{2}, \mathbf{e}_{3}, \mathbf{e}_{4}, \mathbf{e}_{5}, \mathbf{e}_{6}, \mathbf{e}_{7}\right]^{T}=\left(\begin{array}{ccccccc}
0 & 1 & -1 & 0 & 0 & 0 & 0 \\
0 & 0 & 0 & 1 & -1 & 0 & 0 \\
0 & 0 & 0 & 0 & 0 & 1 & -1
\end{array}\right)
$$


At each time step, the entire 3D lattice domain is traversed and for each cell, new distribution function values are computed from its six neighbors (plus itself). The temperature corresponding to the $0^{t h}$ moment is computed as $T(\mathbf{x}, t)=$ $\sum_{i=1}^{7} f_{i}(\mathbf{x}, t)$. Only the data of the cell and its six neighbors are used, so the lattice can be traversed in any order since values from the neighbors are computed from the previous time step. The LBM offers high parallel scalability, second order accuracy in space and the simplicity of an implementation on a uniform Cartesian grid [9]. The relation between LBM equations (Eq. 6, Eq. 7) and the continuous model can be derived by employing the Chapman-Enskog expansion, (a formal multi-scale expansion) [36]. For stability reason, we use a Multiple-Relaxation-Time model since the numerical stability can be improved by separating the relaxation rates of the conserved and non-conserved moments [43]. First, $\mathbf{f}$ is brought to a new basis in which each component corresponds to a certain moment of the vector $\mathbf{M f}\left(0^{t h}\right.$ order is the temperature $T$ ). Then each component relaxes to the equilibrium $\mathbf{M f}^{\mathbf{e q}}$ with a different relaxation coefficient. Finally, the vector is projected back onto the original seven-dimension space [41]. In (Eq. 6), instead of writing $\mathbf{A}=\mathbf{M}^{-1} \hat{\mathbf{S}} \mathbf{M}=1 / \tau \mathbf{I}$, the relaxation towards equilibrium is performed in the moment space, where

$$
\mathbf{M}=\left(\begin{array}{ccccccc}
1 & 1 & 1 & 1 & 1 & 1 & 1 \\
0 & 1 & -1 & 0 & 0 & 0 & 0 \\
0 & 0 & 0 & 1 & -1 & 0 & 0 \\
0 & 0 & 0 & 0 & 0 & 1 & -1 \\
6 & -1 & -1 & -1 & -1 & -1 & -1 \\
0 & 2 & 2 & -1 & -1 & -1 & -1 \\
0 & 0 & 0 & 1 & 1 & -1 & -1
\end{array}\right)
$$

and $\hat{\mathbf{S}}=\operatorname{diag}\left(1,1 / \tau, 1 / \tau, 1 / \tau, 1 / \tau_{1}, 1 / \tau_{1}, 1 / \tau_{1}\right)$ is the collision matrix in moment space. The relaxation time $\tau$ is directly related to the diffusion coefficient $D$ through $\tau=$ $1 / 2+4 D \delta t / \delta x^{2}$ [41]. After a stability analysis, we chose $\tau_{1}=1.33$ [9] to get a stable and well-behaved solution. Based on the Chapman-Enskog expansion, this value does not change the accuracy of the solution but it affects its stability.

No-flux boundary conditions are applied at the border of the liver. The boundaries are treated according to the level set representation of the liver computed from the segmentation using linear interpolation without requiring advanced meshing techniques. We use a second-order accuracy model for curved walls presented by [44] who proposed a simple boundary condition based on interpolation and the bounce back scheme. This method needs to treat the boundary conditions separately for two cases: $\Delta \leq \frac{1}{2}$ or $\Delta>\frac{1}{2}$, i.e either the boundary is closer to the lattice point which is inside the domain or it is closer to the lattice point which is not in the domain. $\Delta$ represents the fraction of an intersected link in the domain of interest (Fig. 5, left for the description of $\Delta$ ) and is computed based on the level-set representation of the liver. If the node from which the post collision values travels $\left(\mathbf{x}-\mathbf{e}_{\alpha} \delta x\right)$ is outside the domain, then the value of $f_{\alpha}(\mathbf{x}, t+\delta t)$ is:

$$
\begin{aligned}
& \text { for } \Delta>\frac{1}{2}: \\
& f_{\alpha}(\mathbf{x}, t+\delta t)=\frac{1}{2 \Delta} \hat{f}_{\bar{\alpha}}(\mathbf{x}, t+\delta t)+\frac{2 \Delta-1}{2 \Delta} \hat{f}_{\alpha}(\mathbf{x}, t+\delta t) \\
& \text { for } \Delta \leq \frac{1}{2}:
\end{aligned}
$$
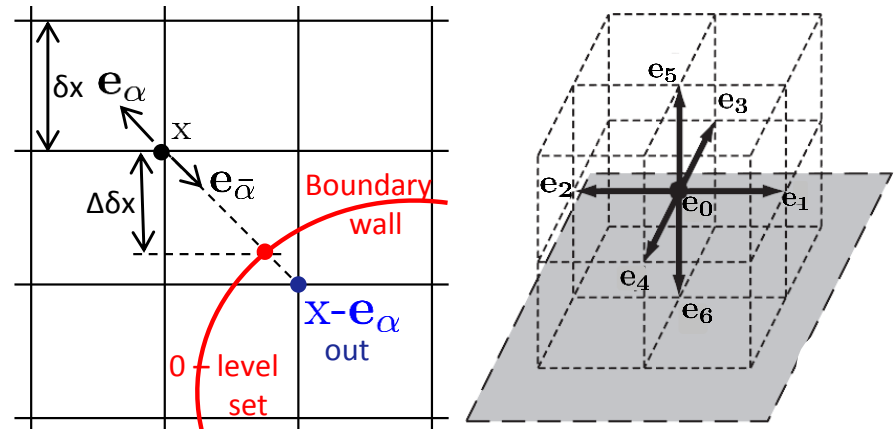

Fig. 5: (Left): Layout of the isotropic Cartesian grid for the implementation of LBM boundary condition with a curved wall boundary. (right): The D3Q7 scheme used from [41].

$$
\begin{aligned}
f_{\alpha}(\mathbf{x}, t+\delta t) & =2 \Delta \hat{f}_{\bar{\alpha}}(\mathbf{x}, t+\delta t) \\
& +(1-2 \Delta) \hat{f}_{\bar{\alpha}}\left(\mathbf{x}-\mathbf{e}_{\bar{\alpha}} \delta x, t+\delta t\right)
\end{aligned}
$$

where $\mathbf{e}_{\bar{\alpha}}=-\mathbf{e}_{\alpha}$.

Finally, we model the heat source term through a Dirichlet boundary condition at the location of the probe. We emulated the RFA protocol by computing a sphere centered at the center of the tumor and with the radius defined pre-operatively by the clinician given the size and the location of the tumor. The temperature of the points inside the sphere is enforced at $105^{\circ} \mathrm{C}$ for a duration defined also pre-operatively.

\section{Model of the Patient Hepatic Venous Circulation System}

Heat transfer in liver tissue is highly dependent on the blood flow circulation. To solve the WK model (Eq. 3), we need the blood velocity field $\mathbf{v}$ everywhere in the parenchyma. The blood inside the liver is modeled as a Newtonian fluid with prespecified density $\rho$ and viscosity $\mu . \mathbf{v}$ is calculated according to Darcy's law [45]:

$$
\mathbf{v}=-\kappa /\left(\mu \epsilon^{2 / 3}\right) \nabla p
$$

where $p$ is the pressure. Because of mass conservation, this amounts to solving the Laplace equation:

$$
\nabla \cdot\left(-\kappa /\left(\mu \epsilon^{2 / 3}\right) \nabla p\right)=0
$$

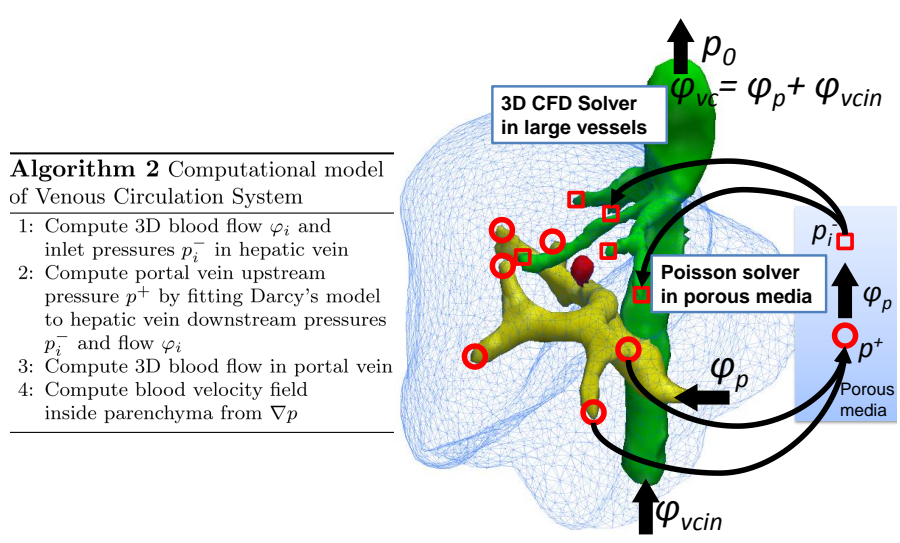

Fig. 6: Model of the hepatic circulatory system. Arrows denote blood flow. Circles and squares denote portal and hepatic vessel tips. See text for details. 
At the border of the liver, Neumann boundary conditions are employed (no flow is leaking). Dirichlet boundary conditions are applied at the tip of the portal and hepatic veins, to define the pressure drop between them. As we cannot estimate these pressures in vivo, we rely on a CFD model of the hepatic venous circulation system to estimate them (Fig. 6). We used a full 3D CFD solver (unsteady incompressible Navier-Stokes equations with viscous terms, expressed in an Eulerian framework which embeds the domain boundary using a level set representation of the segmented vessels [46]). From the segmentation, a tetrahedral multi-domain mesh is generated based on the resulting multi-label mask image using CGAL [47] (www.cgal.org) to compute the porous flow, which is then calculated using FEM on the linear multi-domain tetrahedral mesh. The resulting flow is tri-linearly rasterized on the Cartesian grid after computation. In this work, the effect of heat on the viscosity of the flow is neglected to decouple flowrelated from the heat diffusion calculation for computational efficiency. CFD and porous flow are calculated only once at the beginning of the algorithm.

Let $\varphi_{v c_{i n}}$ be the vena cava inflow, $\varphi_{p}$ the portal vein inflow and $\varphi_{v c}=\varphi_{v c_{i n}}+\varphi_{p}$ the vena cava outflow (conservation of mass, the hepatic artery is neglected in this study but could be added without modification into the framework). We also set the vena cava outlet pressure $p_{0}=3 \mathrm{mmHg}$ in the range of physiological values of healthy patients. The values are listed in Table I. First, we compute the 3D blood flow and pressure distribution within the vena cava and hepatic veins as follows: A plug profile velocity field is applied at the inlets (squares in Fig. 6), computed from the outflow $\varphi_{p}$ and the cross-sectional area of each inlet. The CFD calculation gives the downstream pressures $p_{i}^{-}$and the 3D blood flow $\varphi_{i}$ for each inlet of the hepatic vein. Then we estimate the upstream pressure $p^{+}$, assumed constant, of the portal vein outlets (circles in Fig. 6). We solve Darcy's law and optimize over $p^{+}$such that the computed perfused flow through the hepatic vein inlets $\varphi_{i}$ matches the one computed at the first step using 3D CFD. Then once $p^{+}$is estimated, we compute the blood flow using the 3D CFD solver. Finally, we compute the blood velocity field inside the parenchyma using Eq. 8.

\section{EXPERIMENTS AND RESULTS}

The computer hardware used in all the experiments was a Windows 7 desktop machine (Intel Xeon, $2.80 \mathrm{GHz}, 45 \mathrm{~GB}$ RAM, 24 CPUs) with a Nvidia Quadro 60001.7 GB with 448 CUDA cores.

\section{A. Quantitative Verification of the Heat Transfer Model}

1) Experimental protocol: To evaluate the LBM heat transfer solver, its behavior is compared on a regular cuboid domain with an analytical solution. For a source released at $\mathbf{x}_{0}$ at time $t_{0}$, the $3 \mathrm{D}$ analytical solution of the advection-diffusion equation: $\frac{\partial T}{\partial t}+\mathbf{v} \cdot \nabla T=\nabla \cdot(D \nabla T)$ is:

$T(\mathbf{x}, t)=\frac{M}{\left[4 \pi\left(t-t_{0}\right) D\right]^{3 / 2}} \exp \left(-\frac{\left\|\mathbf{x}-\mathbf{x}_{\mathbf{0}}-\left(t-t_{0}\right) \mathbf{v}\right\|^{2}}{4 D\left(t-t_{0}\right)}\right)$

To have the same conditions as the RFA computation on a patient's liver, parameters were chosen to get the heat diffusion in a physiological range. We set a Gaussian-shape source of $70^{\circ} \mathrm{C}$ at the center of the cuboid at time $t=0$ (Fig. 7) with this set of parameters: $D=0.1 \mathrm{~mm}^{2} / \mathrm{s}, \mathbf{v}=(2,0,0) \mathrm{mm} / \mathrm{s}$, $M=35000^{\circ} \mathrm{C} \cdot \mathrm{mm}^{3}, t_{0}=-50 \mathrm{~s}$. We initialized the temperature values at each point of the domain with the analytical solution at time $t=0$, then the advection-diffusion equation is solved using our LBM solver. The temperature at a typical point of the domain is reported and compared with the analytical solution values. Neumann boundary conditions were used at the border of the domain, which was chosen to be large enough to get rid of the boundary effect at the probed points.

2) LBM Convergence Analysis: We performed a spatial and a temporal convergence analysis of the solution computed by the LBM solver. The solutions are compared to the analytical solution at one typical point of the domain (Fig. 8, left).

a) Spatial Convergence Analysis: First, the time-step is fixed to a constant value and different solutions with different resolutions are computed. As shown in (Fig. 8, left), the smaller the spatial resolution, the closer the computed solution is to the analytical one. Quantitatively, the RootMean-Square (RMS) errors between the computed solution and the analytical one decreased with the resolution: $11.86^{\circ} \mathrm{C}$, $2.42^{\circ} \mathrm{C}, 0.55^{\circ} \mathrm{C}, 0.11^{\circ} \mathrm{C}$ for $5 \mathrm{~mm}, 2 \mathrm{~mm}, 1 \mathrm{~mm}$ and $0.5 \mathrm{~mm}$ respectively. This analysis confirms qualitatively and quantitatively the accuracy of the implementation of the heat transfer model with LBM.

b) Temporal Convergence Analysis: For a given resolution, an upper and lower bound for the time-step were provided by the simulated physics from the LBM implementation and the Courant-Friedrichs-Lewy conditions: $|\mathbf{v} \delta t / \delta x| \leq 1$ [48]. A time-step of $75 \mathrm{~ms}$ and a resolution of $1-2 \mathrm{~mm}$ appeared to be a good compromise between accuracy and computational cost (Fig. 8, right).

\section{B. Computational Efficiency}

A GPU-based version of our model has been developed, relying on the CUDA toolkit (dedicated software for NVIDIA's GPUs) since LBM is easily parallelizable. In our model, the values of interest are the temperature related to the distribution function and the state (alive, vulnerable, or dead) of the cell computed at each vertex separately. This implementation uses classic parallelization methods, similarly to a CPU parallel
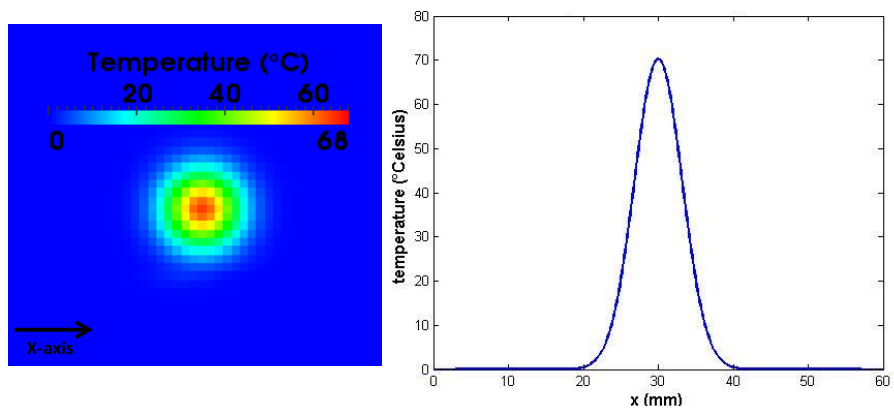

Fig. 7: (Left): Synthetic set-up use in the quantitative verification of the framework at time $\mathrm{t}=0 \mathrm{~s}$. $($ Right $)$ : Initial temperature distribution along the $\mathrm{x}$-axis of the cuboid domain. See text for details. 

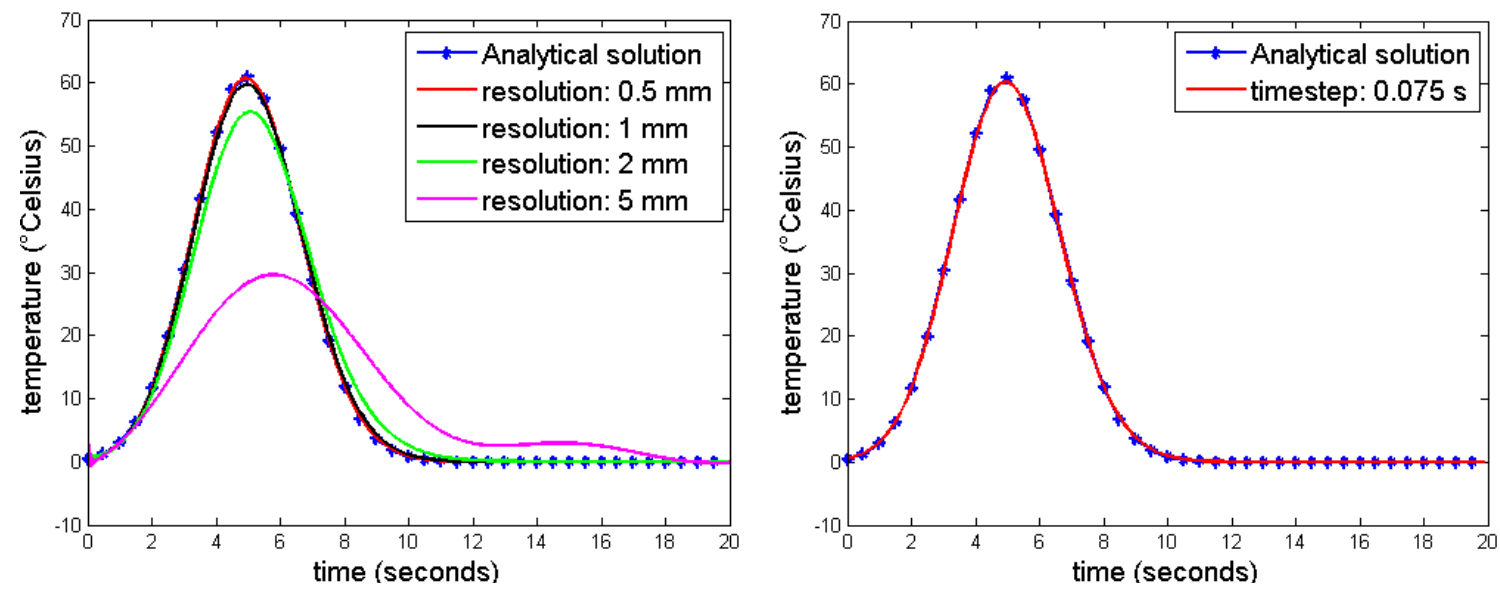

Fig. 8: (Left): Spatial convergence analysis for a fixed time-step of $0.01 s$ for one point in the domain described in Fig. 7. As one can see, the proposed framework quickly converges to the analytical solution of the advection-diffusion equation. (Right): The computed solution for a resolution of $1 \mathrm{~mm}$ and a time-step of $0.075 \mathrm{~s}$ compared to the analytical solution for the same point. The RMS error is reduced to $0.26^{\circ} \mathrm{C}$.

approach: each thread is dedicated to one vertex and computes the contribution of the temperature and state of the cell for this vertex ensuring a tiled access in memory. Two distribution functions are actually needed (at time $\mathrm{t}$ and time $t-1$ : $\mathbf{f}_{\text {past }}$ and $\mathbf{f}_{\text {present }}$ ), to avoid concurrency reading and writing when one thread writes a vertex value while another thread attempts to read it. The implementation is described in Algorithm 2.

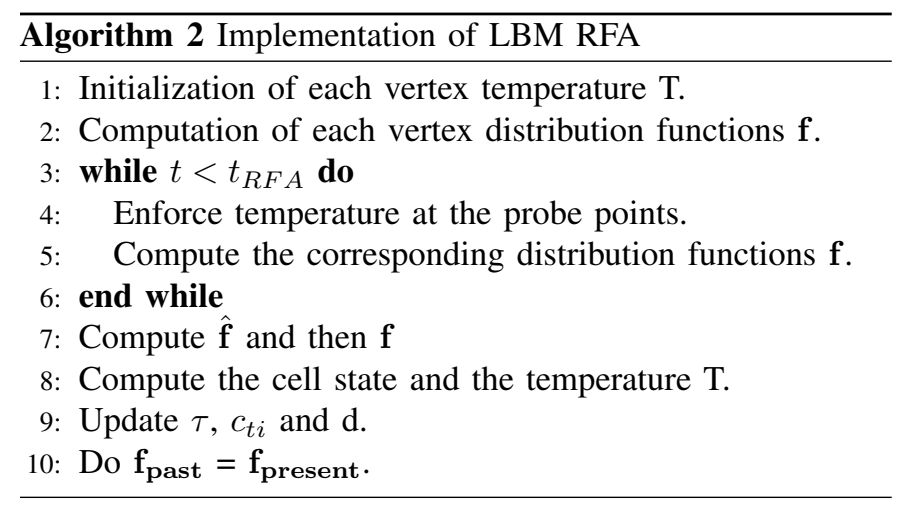

In order to show the benefit of our GPU-based approach, it is compared against a CPU implementation with multithreading. The same synthetic set-up as described in Fig. 7 is used. As reported in Fig. 9, experiments showed a maximum speed-up of 11 with multithreading and 22 threads (OpenMP) and 45 with graphical processing units (GPU) implemented in CUDA with respect to a single-core implementation of LBM. Moreover, after a quantitative verification of the FEM computation against the analytical solution, experiments showed that a $60 \times$ speed-up was obtained with respect to an FEM implementation on CPU for a similar accuracy.

\section{Patient-Specific RFA computation}

1) Experimental Protocol: The model is evaluated on clinical, retrospective data from ten patients, with fourteen ablations (some patient had several tumors ablated) for whom pre- and post-operative CT images were available. For all patients, nominal tissue parameters were employed (Table I). Clinical RFA protocol requires that the probe is deployed within the tumor with a probe diameter defined pre-operatively according to the size of the tumor, and then maintained for 7 minutes after the target temperature of $105^{\circ} \mathrm{C}$ was reached, as measured by the probe thermistors. For large tumors, the process was iterated with sequentially increasing diameters. In all cases, a single probe placement was utilized (no separate overlapping ablations). After anatomical model extraction, we emulated the RFA protocol by placing the virtual probe at the center of the tumor. Cells around the probe tip within the probe diameter sphere were heated at $105^{\circ} \mathrm{C}$ during 7 minutes or twice 7 minutes. In all cases, the computation continued for 3 additional minutes without the probe so that each cell reaches a steady state. In order to evaluate the results of the computation, we compared the computed necrotic region with the patientspecific ground truth. For each patient, the lesion is manually segmented by an expert on the postoperative image and then non-rigidly registered to the preoperative image. The elastic registration from the Advanced Normalization tools (ANTS) is used [49] with the vessels and parenchyma binary images employed as landmarks. Indeed, the thermal induced lesion on the postoperative image creates registration issues, especially
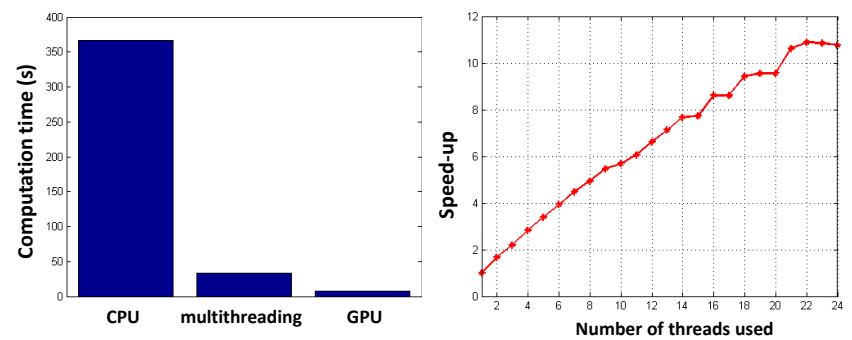

Fig. 9: (Left): Computation time for simple CPU implementation without any parallelization, for multithreading implementation with OpenMP and 22 threads and GPU implementation with CUDA. (Right): Computation speed-up with respect to the number of threads used (parallel optimization with OpenMP). 


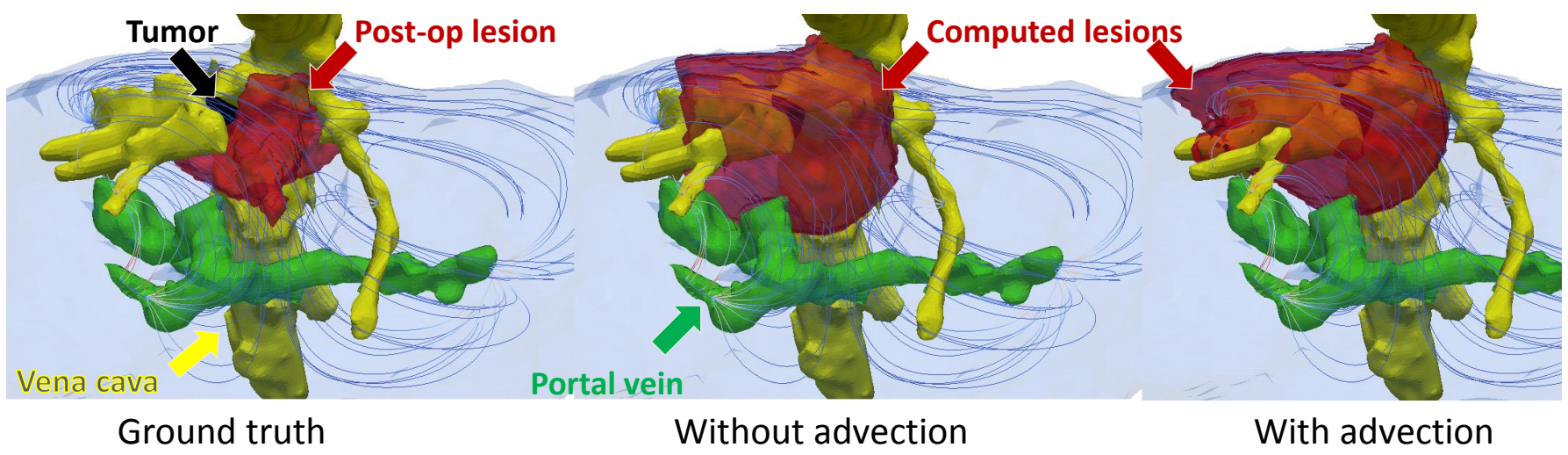

Fig. 10: Results of the computation for patient 04, the streamlines represent the parenchyma flow and are color-coded with respect to the velocity magnitude. As one can see the lesion computed with advection follows the blood flowing from the portal vein to the sushepatic veins. See text for details.

in the area of interest (Fig. 11). Finally the accuracy of the registration is visually checked by the expert.

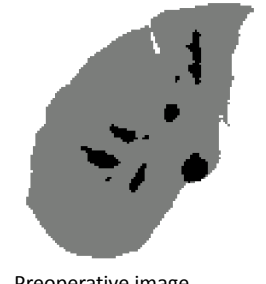

Preoperative image

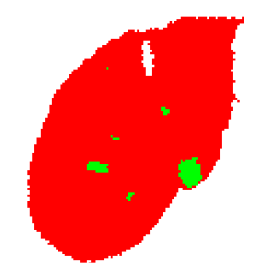

Registered postoperative image

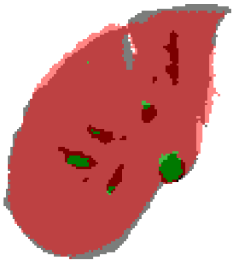

Overlay between preoperative and registered postoperative images
Fig. 11: The registration is performed using masks with vessels and parenchyma as landmarks. The postoperative mask is registered (right) to the preoperative mask (left), with an elastic registration algorithm using ANTS [49]. Presented here are the results for patient 10.

2) Computation Time: One minute of ablation is computed in almost one minute. In comparison, using FEM on CPUs, the identical process takes around one hour. To the best of our knowledge, this is the first time that near real-time physiological computations of RF ablation has been achieved. A single probe ablation of 7 minutes is computed in around 8 minutes depending on the liver size, while an increased probe diameter ablation of 14 minutes is computed in around 15 minutes.

3) Systematic study: Qualitatively, computed ablation followed closely the boundaries of the vessels, due to the heat sink effects of the blood (Fig. 10). The shape of the ablated area is also dependent on the heat advection through the liver parenchyma (Fig. 12). Cell death area computed using the model compared qualitatively well with the observed postoperative necrosis zone as we can see on Fig. 10 and Fig. 12. Quantitatively, in most of the cases, average pointto-mesh errors were within clinical acceptance as they were significantly lower than the different diameter configurations of the probes ( 3 to $5 \mathrm{~cm}$ ), as shown on Table. II. More importantly, in all but one case (patient 01) the computation predicted that the selected protocols completely covered the entire tumor, which is the clinical criterion for ablation planning. The sensitivity is also reasonable (67\% on average).
The average positive Predictive value of $38 \%$ is low as the values are drastically different for each patient (from $95.6 \%$ for patient 02 to $11.9 \%$ for patient 09 ). Some cases presented a computed lesion far from the registered postoperative one. For example, as one can see on Fig. 16, the computed lesion in red is different and far from the registered postoperative lesion in white for patient 03 (PPV of 14.5\%). This may be due to the uncertainty of the actual probe position used in the clinical protocol or to registration errors as discussed in the following section (Sec. IV). Some cases presented a larger necrosis area compared to the ground truth (Fig. 10) or a smaller one (Fig. 17, left). The diffusion coefficient used from the literature was either too high or too low to get a perfect match, as exposed in the following experiment. It may be due to the general state of the liver, which can be cirrhotic or hyperperfused [50].

TABLE II: Evaluation on patient data (PPV: Positive Predictive Value)

\begin{tabular}{|c|l|c|c|c|c|}
\hline Patient & $\begin{array}{l}\text { Probe diam- } \\
\text { eter }(\mathrm{cm})\end{array}$ & $\begin{array}{l}\text { Point-to-mesh } \\
\text { error }(\mathrm{mm})\end{array}$ & $\begin{array}{c}\text { DICE } \\
(\%)\end{array}$ & $\begin{array}{c}\text { Sensitivity } \\
(\%)\end{array}$ & $\begin{array}{l}\text { PPV } \\
(\%)\end{array}$ \\
\hline 01 & 3 & $8.24 \pm 8.48$ & 54.3 & 46.0 & 66.2 \\
\hline 02 & 4 then 5 & $8.25 \pm 5.92$ & 61.7 & 45.6 & 95.6 \\
\hline 03 & 4 & $21.65 \pm 16.96$ & 17.7 & 22.7 & 14.5 \\
\hline 04 & 4 then 5 & $10.91 \pm 8.67$ & 31.4 & 89.5 & 19.0 \\
\hline $05-1$ & 4 & $7.28 \pm 7.03$ & 45.0 & 80.8 & 31.2 \\
$05-2$ & 3.5 & $6.80 \pm 6.25$ & 54.2 & 44.8 & 68.5 \\
\hline 06 & 4 then 5 & $10.77 \pm 5.01$ & 37.6 & 90.7 & 23.7 \\
\hline $07-1$ & 4 then 5 & $8.34 \pm 6.79$ & 40.8 & 66.4 & 29.4 \\
$07-2$ & 4 then 5 & $12.92 \pm 12.32$ & 45.2 & 63.8 & 35.0 \\
$07-3$ & 4 then 5 & $11.61 \pm 11.92$ & 40.3 & 93.6 & 25.7 \\
\hline $08-1$ & 3 & $7.97 \pm 5.41$ & 30.6 & 32.1 & 29.1 \\
$08-2$ & 3 & $5.32 \pm 4.76$ & 61.6 & 65.5 & 58.2 \\
\hline 09 & 3 & $11.96 \pm 8.50$ & 21.3 & 98.9 & 11.9 \\
\hline 10 & 4 then 5 & $10.33 \pm 11.20$ & 44.1 & 96.8 & 28.5 \\
\hline
\end{tabular}

Given $V_{m}$ and $V_{s}$, the volume of measured (respectively simulated) necrotic area, then the DICE score is defined as $D I C E=\frac{2\left|V_{m} \cap V_{s}\right|}{\left|V_{m}\right|+\left|V_{s}\right|}$, the sensitivity is $S=\frac{\left|V_{m} \cap V_{s}\right|}{\left|V_{m}\right|}$ and the Positive Predictive Value is $\frac{\left|V_{m} \cap V_{s}\right|}{\left|V_{s}\right|}$

4) Effect of Advection: In order to better understand the effect of advection, we perform the computations on the same data and we remove the blood flow in the parenchyma. The results are reported in Table III. Qualitatively, we can observe that the advection has an impact on the shape, the extent and 

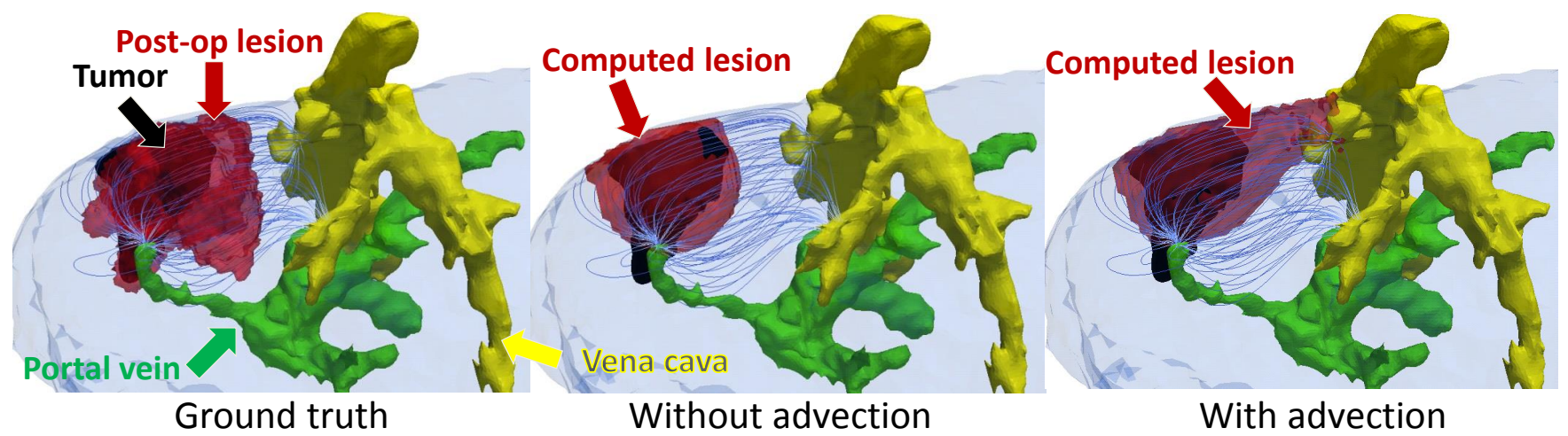

Fig. 12: Results of the computation for patient 01 , the streamlines represent the parenchyma flow and are color-coded with respect to the velocity magnitude. As one can see the lesion computed with advection is closer to the ground truth as it follows the blood flow path from one inlet of the portal vein to the vena cava. See text for details.

the size of the lesion (Fig. 10 and Fig. 12). As the blood flow in the parenchyma tends to go from the extremities of the portal vein to the hepatic veins (the extremities of the vena cava), the temperature follows the same path. Notably, if the tumor is close to the portal vein, the advection will tend to enlarge the extent of the necrotic region as the blood flow will evacuate the temperature inside the liver parenchyma, whereas if the tumor is closer to the vena cava, the advection will tend to reduce the size of the necrotic core as the temperature will be dissipated in the general blood flow vasculature. Therefore, we can claim that the effect of the advection is roughly proportional to the signed geodesic distance between the vena cava and the portal vein. Thus the impact of parenchyma perfusion is highly dependent on the tumor location.

TABLE III: Evaluation on patient data without advection

\begin{tabular}{|c|c|c|c|c|}
\hline Patient & $\begin{array}{c}\text { Point-to-mesh } \\
\text { error }(\mathrm{mm})\end{array}$ & $\begin{array}{c}\text { DICE } \\
(\%)\end{array}$ & $\begin{array}{c}\text { Sensitivity } \\
(\%)\end{array}$ & $\begin{array}{c}\text { PPV } \\
(\%)\end{array}$ \\
\hline 01 & $9.03 \pm 9.74$ & 52.4 & 43.7 & 65.3 \\
\hline 02 & $8.00 \pm 5.01$ & 64.6 & 48.9 & 95.2 \\
\hline 03 & $24.44 \pm 20.91$ & 18.3 & 22.4 & 15.5 \\
\hline 04 & $10.57 \pm 5.59$ & 28.8 & 96.6 & 16.9 \\
\hline $05-1$ & $5.56 \pm 5.20$ & 51.7 & 83.8 & 37.4 \\
$05-2$ & $6.19 \pm 4.56$ & 54.5 & 59.8 & 50.1 \\
\hline 06 & $8.25 \pm 5.92$ & 37.9 & 82.6 & 24.6 \\
\hline $07-1$ & $6.06 \pm 3.99$ & 55.3 & 79.7 & 42.3 \\
$07-2$ & $7.98 \pm 5.05$ & 56.8 & 71.2 & 47.2 \\
$07-3$ & $4.73 \pm 3.30$ & 67.8 & 87.9 & 55.2 \\
\hline $08-1$ & $6.70 \pm 4.43$ & 38.8 & 51.6 & 31.0 \\
$08-2$ & $5.07 \pm 4.45$ & 60.5 & 61.3 & 59.7 \\
\hline 09 & $6.91 \pm 4.65$ & 33.0 & 97.9 & 19.9 \\
\hline 10 & $6.76 \pm 4.08$ & 51.5 & 98.8 & 34.8 \\
\hline
\end{tabular}

5) Effect of The Probe Position: The position of the probe is a major unknown in our experiments. We assumed that the clinician put the probe at the center of the tumor (Fig. 13, left) but it may not always be the case. To check the sensitivity of the computations to the probe position, a new computation is performed for patient 06. The same configuration was used except that the probe center is at the barycenter of the registered post-op lesion (Fig. 13, right), not anymore at the center of the tumor. With this technique, the results are significantly improved (Table. IV). The probe position affects the error measured between the computed necrotic area and

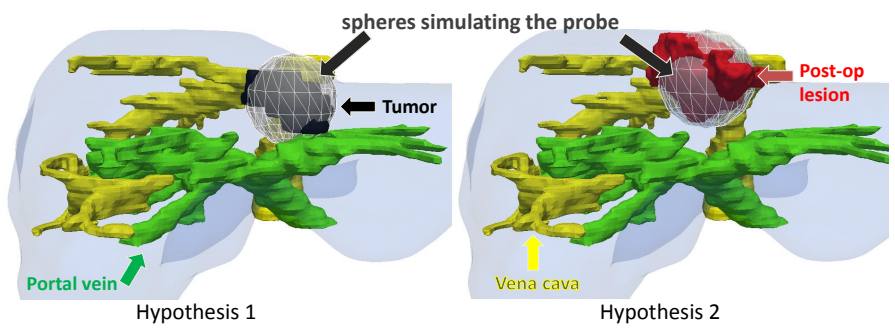

Fig. 13: The two different hypotheses used for the probe placement. (Hypothesis 1): The probe is placed at the center of the tumor $=$ regular hypothesis. (Hypothesis 2): The probe is placed at the center of the post-op registered lesion (patient 06).

TABLE IV: Evaluation of the effect of the probe placement with the two hypotheses decreased in Fig. 13

\begin{tabular}{|l|c|c|c|c|}
\hline Patient 06 & Point-to-mesh error & DICE & Sensitivity & PPV \\
\hline Hypothesis 1 & $10.77 \pm 5.01 \mathrm{~mm}$ & $37.6 \%$ & $90.7 \%$ & $23.7 \%$ \\
\hline Hypothesis 2 & $8.01 \pm 6.50 \mathrm{~mm}$ & $50.0 \%$ & $96.3 \%$ & $33.8 \%$ \\
\hline
\end{tabular}

the ground truth (Fig. 14).
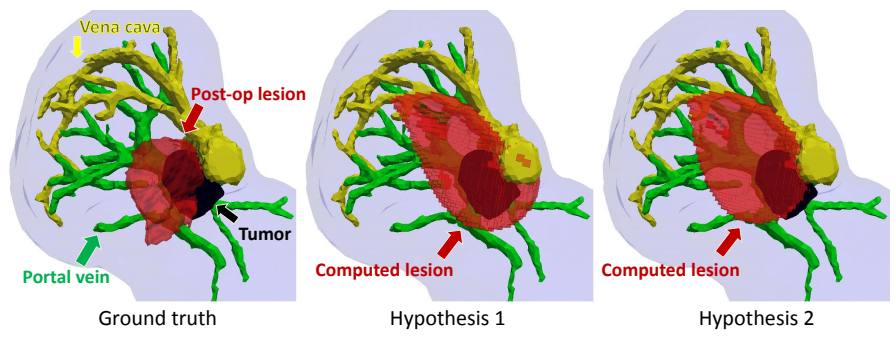

Fig. 14: The different results in red with the different hypotheses are presented. (Left): Postoperative lesion registered to the preoperative image. (Middle): Computed lesion with the first hypothesis. (Right): Computed lesion with the second hypothesis. The boundaries of second lesion is closer the one of the ground truth (patient 06).

6) Effect of The Segmentation: The segmentation also has an influence on the computed lesion (Table. V). To notice the effect of user variability on the segmentation, the expert segmented twice the data of patient 03 . This second segmentation was done independently and weeks apart from the first 


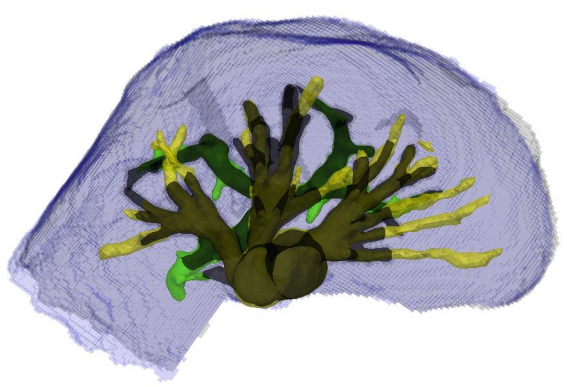

2 segmentations of the same patient

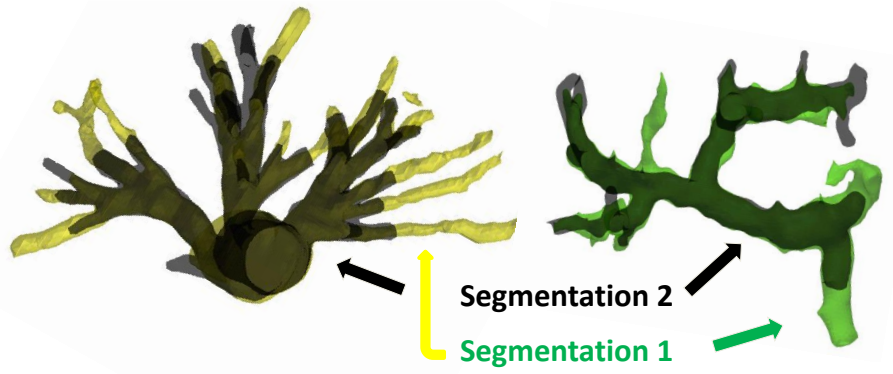

Vena cava

Portal vein

Fig. 15: Two segmentations of the same patient performed by the same expert several weeks apart. The parenchyma boundaries are similar, but the vessels segmentation differ greatly from one segmentation to the other.

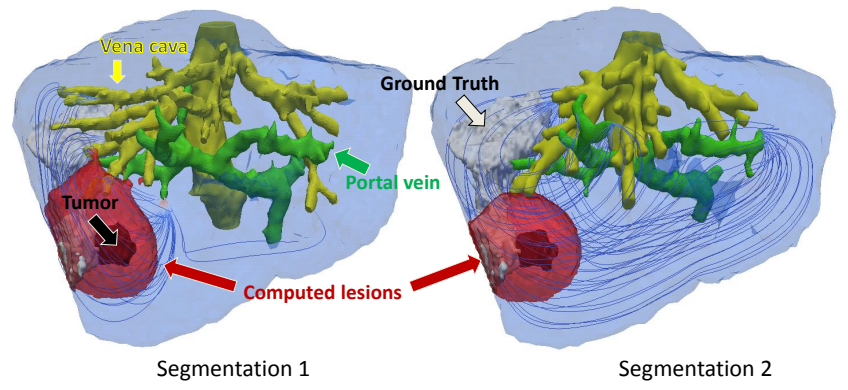

Fig. 16: The two computed lesions for patient 03, the streamlines represent the parenchyma flow and are color-coded with respect to the velocity magnitude. The total blood flow is the liver is the same (around $25 \mathrm{~mL} / \mathrm{s}$ ) but the parenchyma blood does not flow similarly in both cases due to the differences in the extremities segmentations.

one. Fig 15 shows the superposition of the two segmentations. Based on these two segmented datasets, we computed twice the RFA ablation and compared the two computed lesions (Fig 16). It can be noted that the main difference between the two computations lies in the CFD results which is really sensitive to the segmentation, especially the segmentation of the vessels inlets.

TABLE V: Evaluation of the effect of the segmentation

\begin{tabular}{|l|c|c|c|c|}
\hline Patient 03 & Point-to-mesh error & DICE & Sensitivity & PPV \\
\hline Segmentation 1 & $21.65 \pm 16.96 \mathrm{~mm}$ & $17.7 \%$ & $22.7 \%$ & $14.5 \%$ \\
\hline Segmentation 2 & $19.07 \pm 17.11 \mathrm{~mm}$ & $22.8 \%$ & $28.4 \%$ & $19.0 \%$ \\
\hline
\end{tabular}

7) Effect of biophysical parameters: The accuracy of the computation depends on the material properties since they are patient-specific, temperature- and space-dependent and they cannot be easily measured in vivo. Nominal tissue parameters were employed, with values often based on ex vivo experiments on animal tissue sometimes with a large varying range between published studies [51]. A proper estimation of those parameters is needed but has often been overlooked due to its difficulty. A first step towards the personalization of those parameters is to perform a sensitivity study of the computation to the main parameters. From the results based on nominal parameters for patient 02 , we can see that the heat conductivity is too low. For a heat conductivity 8 times greater than the nominal value $\left(4.096 W(m K)^{-1}\right)$, we manage to have a better outcome (Fig. 17). Quantitatively, Table VI shows improvements in the point-to-mesh error (from $8.25 \pm 5.92$ $\mathrm{mm}$ to $3.85 \pm 3.21 \mathrm{~mm}$ ) and in the Dice index (from $61.7 \%$ to $82.3 \%$ ).

TABLE VI: Estimation of the heat conductivity (PPV: Positive Predictive Value)

\begin{tabular}{|c|c|c|c|c|}
\hline $\begin{array}{l}\text { Patient 02 } \\
\text { Probe diameter: } \\
4 \mathrm{~cm} \text { then 5 cm }\end{array}$ & $\begin{array}{l}\text { Point-to-mesh } \\
\text { error }(\mathrm{mm})\end{array}$ & $\begin{array}{c}\text { DICE } \\
(\%)\end{array}$ & $\begin{array}{c}\text { Sensitivity } \\
(\%)\end{array}$ & $\begin{array}{c}\text { PPV } \\
(\%)\end{array}$ \\
\hline $\mathrm{D}$ & $8.25 \pm 5.92$ & 61.7 & 45.6 & 95.6 \\
\hline $\mathrm{D} \times 2$ & $6.97 \pm 5.26$ & 68.2 & 54.1 & 92.2 \\
\hline $\mathrm{D} \times 4$ & $5.17 \pm 4.07$ & 76.3 & 67.3 & 88.0 \\
\hline $\mathrm{D} \times 6$ & $4.17 \pm 3.38$ & 80.8 & 77.5 & 84.4 \\
\hline $\mathrm{D} \times 8$ & $3.85 \pm 3.21$ & 82.3 & 84.8 & 80.1 \\
\hline $\mathrm{D} \times 10$ & $4.15 \pm 3.38$ & 81.3 & 89.3 & 74.6 \\
\hline $\mathrm{D} \times 12$ & $4.88 \pm 3.87$ & 78.6 & 92.0 & 68.6 \\
\hline $\mathrm{D} \times 14$ & $5.83 \pm 4.53$ & 75.0 & 93.6 & 62.6 \\
\hline
\end{tabular}

\section{DISCUSSION}

We have presented a multi-physics model for efficient patient-specific planning of RFA based on medical CT images. We rely on LBM to solve the bioheat equations and the level set representations of the structures are directly computed from images, but our framework still requires advanced meshing techniques to compute the flow in porous media. Despite possible biases in establishing correspondences between the postto the pre-operative images due to registration or segmentation errors, and the use of nominal biological parameters, which are not patient-specific, our model provided promising results, opening new opportunities for RFA planning and guidance.

\section{A. Model Limitations}

In this study, a two-compartment model was used to describe the heat propagation, coming from two simplifications of the coupled bio-heat equations which are accurate in the cases of small and large vessels respectively, whereas previous studies suggested that heat dissipation may arise precisely in the medium vessels [1]. This simplification was motivated by the fact that the liver is highly vascularized: modeling the impact of all vessels is not practical in clinical practice. 


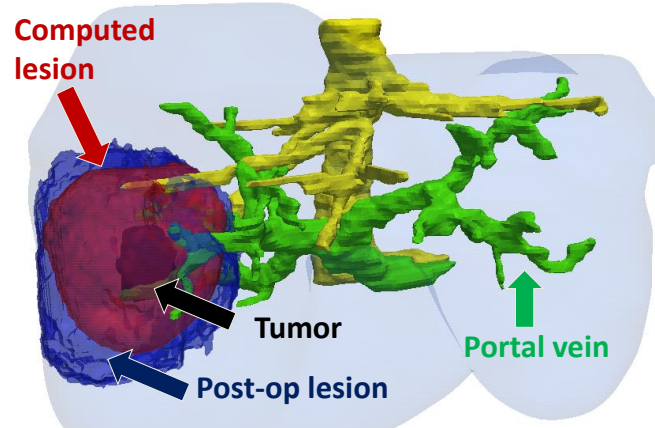

Simulation with nominal parameters

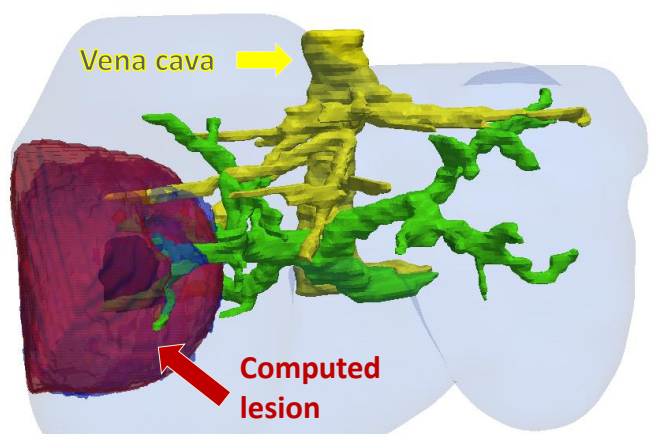

Simulation with personalized parameters

Fig. 17: (Left): computed lesion in red is smaller than the post-op lesion in blue. (Right): A better match is achieved between the computed lesion and the ground truth with adjusted heat conductivity for the same patient (patient 02).

\section{B. Effect of large vesssels}

The computed lesions show that large vessels act as heat sinks as the lesion follows the large vessel's walls. A generic vena cava pressure, inflow and flow through the inlets of the hepatic veins were used for all computations. Since these values were not available, we assumed the pressure and the flow to be a constant in the physiological range of healthy patients. Yet, the pressure and the flow should be personalized as it can vary from patient to patient but also spatially and temporally inside the liver. And the blood flow should instead be modeled as a pulsatile flux. These parameters could be adjusted based on catheter or imaging information such as Phase-Contrast MRI, which can measure the flow in the visible vessels. This study was performed with a weak coupling of heat propagation and CFD. Change in tissue viscosity was neglected as we compute the blood flow only once, at the beginning and then we focused on the heat propagation and cellular necrosis only. A fully coupled computation where the tissue viscosity and coagulation are considered could improve the outcome of the RFA model, i.e the extent of ablation and may provide insights on the entire physical mechanisms involved in RFA and tissue properties for long-term therapy prognosis. This aspect of RFA modeling will be investigated in future work.

\section{Effect of Advection}

The results of the fourteen ablations computed with the complete model and without the advection term demonstrate that the perfusion in the parenchyma does have an effect on the shape and the extent of the thermal induced lesion, depending on the location of the tumor. Therefore the perfusion in the liver may be an important factor to quantify the extent of ablation and predict therapies. Recent experimental studies on pig livers show an increase in the thermal conductivity with decreased distance to large blood vessels and with the perfusion rate and significant directional differences in thermal conductivity [52]. In this study, nominal values were used for the advection coefficient, which control the extent of the heat transport. Yet, this parameter could be estimated and animal data could help to better understand this phenomenon [53] using MRI-thermometry acquired intra-operatively for example.

\section{Effect of the Probe Position}

We showed that the probe position is an important unknown and the RFA computation could be improved by adding this information. As the probe insertion during the RF ablation is done under ultrasound guidance, 3D ultrasound images could be used to know the exact position of the probe. Additionally, the simulation of the probe using Dirichlet boundary condition is simplistic, and a geometrical model describing the exact shape of the probe would probably be beneficial for this framework.

\section{E. Effect of the segmentation}

We saw that the advection and large vessels may have an important effect on the size, shape, and extent of the lesion. More extensive sensitivity analysis of this effect with respect to the segmentation of the vessels should be performed. Modification of the reconstructed anatomy, in particular in the circulation system tree may have an important impact on the parenchyma blood flow and therefore on the advection influence, as shown for patient 03 on Fig. 16.

In this study, as only late venous phase CT images were available, we could not segment the hepatic arteries, thus the effects of the arterial flow were not considered to predict the extent of ablation. Yet, it is worth noting that the veins account for more than $70 \%$ of the blood inflow of the liver parenchyma [54]. A complete study would benefit from including the hepatic artery inflow, accounting for about one fifth of the hepatic blood inflow, but which comes at a higher pressure and pulsation characteristics compared to the portal vein inflow. If the three phases (arterial, portal and venous) CT images were available, it would be straightforward to include hepatic arteries as well as more venous vessels without any modifications for improved accuracy, our framework being modular.

\section{F. Towards personalization}

Finally, tissue properties in the liver are spatially-varying and may also vary with the disease-state of the liver (e.g cirrhosis), but they are difficult to estimate from clinical data since they cannot be quantified from CT imaging for instance. 
In this study, all patients had normal liver parenchyma without cirrhosis or steatosis and heat propagation was modeled with standard tissue parameters reported in the literature for all patients. However our method makes it possible to estimate global patient-specific tissue parameters, which may increase the accuracy of the computation as is the case in adjusting the heat conductivity for one patient in this study. Moreover, the perfusion of the tumor was not taken into account which can impact its thermal conductivity. Yet, the border zone beyond the margin of the tumor is of greater interest and is considered as regular hepatic tissue. Further investigations and experiments on patient-specific tissue parameters will be necessary to include these new findings into our model.

Preliminary experiments suggest that systematic sensitivity analysis and personalized computations with patient-specific and not nominal parameters are necessary to get a better match with the actual outcome [55].

\section{CONCLUSiOns}

We have presented an integrated framework for fast modeling of RFA, which compute and predict RFA intervention outcome in terms of necrotic zone. To the best of our knowledge, it constitutes the largest and most comprehensible in vivo study reported so far. This paper presents novel contributions in RFA computational simulation, which can be summarized as follows:

1) A complete patient-specific geometry including hepatic venous circulation system;

2) The patient-specific modeling including simultaneously the cooling effect of large vessels and of the perfusion within the parenchyma;

3) The discretization method (LBM), which is fast and verified against an analytical solution;

4) Validation on a dataset of ten patients;

5) Experiments testing several hypotheses in the discussion.

Turning computational models of RFA into clinical practice is a challenging task, which can be beneficial for therapy understanding. We focused on modeling heat propagation and cellular necrosis based on a patient image while considering the heat sink effect of blood vessels and porous circulation in the liver. Through the use of the Lattice Boltzmann Method, our system allows near real time and state of the art computations of heat transfer that are suitable for model-based therapy planning or guidance in the future, even if the target is to go beyond real-time, as we need to stay under 1-2 minutes of computation time for clinical use. Based on our approach, the clinician could test different therapeutical strategies insilico, assess their outcome before the intervention and finally choose the most appropriate therapy for a specific patient. A necessary step before deploying this method in clinical settings is a pre-clinical validation with extensive data on larger populations to evaluate the computational model of RFA. This will be possible due to the level of integration of the proposed framework. Our system may thus constitute a first step towards clinical application of a RFA computational model. From a research point of view, the proposed framework paves the way to the quantitative and systematic evaluation of computational models of RFA. There is growing evidence that patient-specific anatomical models are necessary to fully comprehend RFA treatments, in particular in the light of recent in vivo studies [1]. By providing a complete system for patientspecific modeling, our system may be used as input to more comprehensive inverse problem studies, and constitute a useful surrogate tool for RFA planning, potentially improving the outcomes for the patients.

\section{ACKNOWLEDGMENT}

Part of this work was funded by the European Research Council through the ERC Advanced Grant MedYMA 2011291080 (on Biophysical Modeling and Analysis of Dynamic Medical Images).

\section{REFERENCES}

[1] H. B. El-Serag, J. A. Davila, N. J. Petersen, and K. A. McGlynn, "The continuing increase in the incidence of hepatocellular carcinoma in the united states: An update," Ann Intern Med, vol. 139, no. 10, pp. 817 $823,2003$.

[2] S. Garrean, J. Hering, A. Saied, W. S. Helton, and N. J. Espat, "Radiofrequency ablation of primary and metastatic liver tumors: a critical review of the literature," The American Journal of Surgery, vol. 195, no. 4, pp. 508-520, 2008.

[3] P. Hildebrand, T. Leibecke, M. Kleemann, L. Mirow, M. Birth, H. Bruch and C. Bürk, "Influence of operator experience in radiofrequency ablation of malignant liver tumours on treatment outcome," European Journal of Surgical Oncology (EJSO), vol. 32, no. 4, pp. 430-434, 2006.

[4] Y.-s. Kim, H. Rhim, O. K. Cho, B. H. Koh, and Y. Kim, "Intrahepatic recurrence after percutaneous radiofrequency ablation of hepatocellular carcinoma: analysis of the pattern and risk factors," European journal of radiology, vol. 59, no. 3, pp. 432-441, 2006.

[5] X. Chen and G. M. Saidel, "Mathematical modeling of thermal ablation in tissue surrounding a large vessel," J Biomech, vol. 131, 2009.

[6] Y. Jiang, S. Mulier, W. Chong, M. Diel Rambo, F. Chen, G. Marchal, and Y. Ni, "Formulation of 3D finite elements for hepatic radiofrequency ablation," IJMIC, vol. 9, no. 3, pp. 225-235, 2010.

[7] T. Kröger, T. Pätz, I. Altrogge, A. Schenk, K. Lehmann, B. Frericks, J. Ritz, H. Peitgen, and T. Preusser, "Fast estimation of the vascular cooling in RFA based on numerical simulation," Open Biomed Eng J, vol. 4, pp. 16-26, 2010.

[8] C. Rieder, T. Kroeger, C. Schumann, and H. K. Hahn, "GPU-based realtime approximation of the ablation zone for radiofrequency ablation," Visualization and Computer Graphics, IEEE Transactions on, vol. 17, no. 12, pp. 1812-1821, 2011.

[9] S. Rapaka, T. Mansi, B. Georgescu, M. Pop, G. Wright, A. Kamen, and D. Comaniciu, "LBM-EP: Lattice-Boltzmann method for fast cardiac electrophysiology simulation from 3D images," in MICCAI 2012, ser. LNCS, vol. 7511, 2012, pp. 33-40.

[10] S. Tungjitkusolmun, S. T. Staelin, D. Haemmerich, J.-Z. Tsai, H. Cao, J. G. Webster, F. T. Lee Jr, D. M. Mahvi, and V. R. Vorperian, "Threedimensional finite-element analyses for radio-frequency hepatic tumor ablation," Biomedical Engineering, IEEE Transactions on, vol. 49, no. 1 pp. 3-9, 2002.

[11] D. Panescu, J. G. Whayne, S. D. Fleischman, M. S. Mirotznik, D. K. Swanson, and J. G. Webster, "Three-dimensional finite element analysis of current density and temperature distributions during radio-frequency ablation," Biomedical Engineering, IEEE Transactions on, vol. 42, no. 9, pp. 879-890, 1995.

[12] J. D. Doss, "Calculation of electric fields in conductive media," Medical physics, vol. 9, no. 4, pp. 566-573, 1982.

[13] R. Khlebnikov and J. Muehl, "Effects of needle placement inaccuracies in hepatic radiofrequency tumor ablation," in Engineering in Medicine and Biology Society (EMBC), 2010 Annual International Conference of the IEEE. IEEE, 2010, pp. 716-721.

[14] W. Schramm, D. Yang, and D. Haemmerich, "Contribution of direct heating, thermal conduction and perfusion during radiofrequency and microwave ablation," in Engineering in Medicine and Biology Society, 2006. EMBS'06. 28th Annual International Conference of the IEEE. IEEE, 2006, pp. 5013-5016. 
[15] S. Payne, R. Flanagan, M. Pollari, T. Alhonnoro, C. Bost, D. O’Neill, T. Peng, and P. Stiegler, "Image-based multi-scale modelling and validation of radio-frequency ablation in liver tumours," Philos T Roy Soc A, vol. 369, no. 1954, pp. 4233-4254, 2011.

[16] A. Nakayama and F. Kuwahara, "A general bioheat transfer model based on the theory of porous media," International Journal of Heat and Mass Transfer, vol. 51, no. 1112, pp. 3190 - 3199, 2008.

[17] W. Roetzel and Y. Xuan, "Transient response of the human limb to an external stimulust," Internal journal of heat and mass transfer, vol. 41, no. 1, pp. 229-239, 1998.

[18] A.-R. Khaled and K. Vafai, "The role of porous media in modeling flow and heat transfer in biological tissues," Internal journal of heat and mass transfer, vol. 46, no. 26, pp. 4989-5003, 2003.

[19] M. S. Akira Nakayama, Fujio Kuwahara and G. Xu, "A two-energy equation model for conduction and convection in porous media," International Journal of Heat and Mass Transfer, vol. 44, no. 22, pp. 4375 $-4379,2001$.

[20] D. Shrivastava and J. T. Vaughan, "A generic bioheat transfer thermal model for a perfused tissue," Journal of biomechanical engineering, vol. 131, no. 7, p. 074506, 2009.

[21] S. Mahjoob and K. Vafai, "Analytical characterization and production of an isothermal surface for biological and electronic applications," Journal of Heat Transfer, vol. 131, no. 5, p. 52604, 2009.

[22] S. Labonté, "Numerical model for radio-frequency ablation of the endocardium and its experimental validation," Biomedical Engineering, IEEE Transactions on, vol. 41, no. 2, pp. 108-115, 1994.

[23] J. Chato, "Heat transfer to blood vessels." Journal of Biomechanical Engineering, vol. 102, no. 2, pp. 110-118, 1980.

[24] J. W. Mitchell and G. E. Myers, "An analytical model of the countercurrent heat exchange phenomena," Biophysical journal, vol. 8, no. 8, pp. 897-911, 1968 .

[25] H. H. Pennes, "Analysis of tissue and arterial blood temperatures in the resting human forearm," J Appl Physiol, vol. 85, no. 1, pp. 5-34, 1998.

[26] T. Peng, D. ONeill, and S. Payne, "A two-equation coupled system for determination of liver tissue temperature during thermal ablation," International Journal of Heat and Mass Transfer, vol. 54, no. 9, pp. 2100-2109, 2011.

[27] C. A. Linte, J. J. Camp, D. R. Holmes III, M. E. Rettmann, and R. A. Robb, "Toward online modeling for lesion visualization and monitoring in cardiac ablation therapy," in Medical Image Computing and Computer-Assisted Intervention-MICCAI 2013. Springer, 2013, pp. 9-17.

[28] H. Talbot, M. Lekkal, R. Béssard-Duparc, S. Cotin et al., "Interactive planning of cryotherapy using physically-based simulation," in MMVR 21-Medicine Meets Virtual Reality-2014, 2014.

[29] H. Klinger, "Heat transfer in perfused biological tissue I: General theory," B Math Biol, vol. 36, no. 0, pp. 403 - 415, 1974.

[30] W. Wulff, "The energy conservation equation for living tissue," Biomedical Engineering, IEEE Transactions on, no. 6, pp. 494-495, 1974.

[31] E. J. Berjano, "Theoretical modeling for radiofrequency ablation: stateof-the-art and challenges for the future," Biomedical engineering online, vol. 5, no. 1, p. 24, 2006.

[32] K. Diller, J. Oden, C. Bajaj, J. Browne, J. Hazle, I. Babuska, J. Bass, L. Bidaut, L. Demkowicz, A. Elliott et al., "Computational infrastructure for the real-time patient-specific treatment of cancer," Advances in Numerical Heat Transfer, vol. 3, 2008.

[33] D. ONeill, T. Peng, P. Stiegler, U. Mayrhauser, S. Koestenbauer, K. Tscheliessnigg, and S. Payne, "A three-state mathematical model of hyperthermic cell death," Ann Biomed Eng, vol. 39, pp. 570-579, 2011.

[34] F. Henriques Jr and A. Moritz, "Studies of thermal injury: I. the conduction of heat to and through skin and the temperatures attained therein. a theoretical and an experimental investigation," The American journal of pathology, vol. 23, no. 4, p. 530, 1947.

[35] A. R. Moritz and F. Henriques Jr, "Studies of thermal injury: II. the relative importance of time and surface temperature in the causation of cutaneous burns," The American journal of pathology, vol. 23, no. 5, p. 695, 1947.

[36] S. Chen and G. D. Doolen, "Lattice Boltzmann method for fluid flows," Annual review of fluid mechanics, vol. 30, no. 1, pp. 329-364, 1998.

[37] C. Audigier, T. Mansi, H. Delingette, S. Rapaka, V. Mihalef, P. Sharma, A. Kamen, D. Carnegie, E. Boctor, M. Choti et al., "Lattice-Boltzmann method for fast patient-specific simulation of liver tumor ablation from CT images," in Medical Image Computing and Computer-Assisted Intervention-MICCAI 2013. Springer, 2013, pp. 323-330.

[38] A. Criminisi, T. Sharp, and A. Blake, "Geos: Geodesic image segmentation," in Computer Vision-ECCV 2008. Springer, 2008, pp. 99-112.
[39] P. A. Yushkevich, J. Piven, H. Cody Hazlett, R. Gimpel Smith, S. Ho, J. C. Gee, and G. Gerig, "User-guided 3D active contour segmentation of anatomical structures: Significantly improved efficiency and reliability," Neuroimage, vol. 31, no. 3, pp. 1116-1128, 2006.

[40] H.-C. Mai, K.-H. Lin, C.-H. Yang, and C.-A. Lin, "A thermal lattice Boltzmann model for flows with viscous heat dissipation," Computer Modeling in Engineering \& Sciences(CMES), vol. 61, no. 1, pp. 45-62, 2010.

[41] H. Yoshida and M. Nagaoka, "Multiple-relaxation-time lattice Boltzmann model for the convection and anisotropic diffusion equation," $J$ Comput Phys, vol. 229, no. 20, pp. 7774 - 7795, 2010.

[42] S. P. Dawson, S. Chen, and G. Doolen, "Lattice Boltzmann computations for reaction-diffusion equations," The Journal of Chemical Physics, vol. 98, no. 2, pp. 1514-1523, 1993.

[43] D. d'Humières, "Multiple-relaxation-time lattice Boltzmann models in three dimensions," Philosophical Transactions of the Royal Society of London. Series A: Mathematical, Physical and Engineering Sciences, vol. 360, no. 1792, pp. 437-451, 2002.

[44] M. Bouzidi, M. Firdaouss, and P. Lallemand, "Momentum transfer of a Boltzmann-lattice fluid with boundaries," Physics of Fluids, vol. 13, p. 3452, 2001.

[45] H. Brinkman, "A calculation of the viscous force exerted by a flowing fluid on a dense swarm of particles," Applied Scientific Research, vol. 1, no. 1, pp. 27-34, 1949.

[46] K. Ralovich, L. Itu, V. Mihalef, P. Sharma, R. Ionasec, D. Vitanovski, W. Krawtschuk, A. Everett, R. Ringel, and N. Navab, "Hemodynamic assessment of pre- and post-operative aortic coarctation from MRI," in MICCAI 2012, ser. LNCS. Springer, 2012, vol. 7511, pp. 486-493.

[47] P. Alliez, L. Rineau, S. Tayeb, J. Tournois, and M. Yvinec, "3D mesh generation," CGAL User and Reference Manual, vol. 3.9, 2011.

[48] R. Courant, K. Friedrichs, and H. Lewy, "Über die partiellen differenzengleichungen der mathematischen physik," Mathematische Annalen, vol. 100, no. 1, pp. 32-74, 1928.

[49] B. B. Avants, N. Tustison, and G. Song, "Advanced normalization tools (ANTS)," Insight J, 2009.

[50] B. E. Van Beers, I. Leconte, R. Materne, A. M. Smith, J. Jamart, and Y. Horsmans, "Hepatic perfusion parameters in chronic liver disease: dynamic CT measurements correlated with disease severity," American Journal of Roentgenology, vol. 176, no. 3, pp. 667-673, 2001.

[51] H. Tiesler, "Identification of material parameters from temperature measurements in radio frequency ablation," $\mathrm{Ph}$.D. dissertation, $\mathrm{PhD}$ thesis, University of Bremen, 2011, http://elib. suub. uni-bremen. de/peid, 2011.

[52] R. J. Podhajsky, M. Yi, and R. L. Mahajan, "Differential and directional effects of perfusion on electrical and thermal conductivities in liver," in Engineering in Medicine and Biology Society, 2009. EMBC 2009. Annual International Conference of the IEEE. IEEE, 2009, pp. 42954298.

[53] H.-L. M. Cheng and D. B. Plewes, "Tissue thermal conductivity by magnetic resonance thermometry and focused ultrasound heating," Journal of Magnetic Resonance Imaging, vol. 16, no. 5, pp. 598-609, 2002.

[54] W. G. Schenk Jr, J. C. McDonald, K. McDonald, and T. Drapanas, "Direct measurement of hepatic blood flow in surgical patients: with related observations on hepatic flow dynamics in experimental animals," Ann Surg, vol. 156, no. 3, pp. 463-469, 1962.

[55] C. Audigier, T. Mansi, H. Delingette, S. Rapaka, V. Mihalef, D. Carnegie, E. Boctor, M. Choti, A. Kamen, D. Comaniciu et al., "Parameter estimation for personalization of liver tumor radiofrequency ablation," in MICCAI Workshop on Abdominal Imaging-Computational and Clinical Applications, 2014. 